\title{
On the Distribution of Dust in the Large Magellanic Cloud ${ }^{1}$
}

\author{
Jason Harris, Dennis Zaritsky, \\ UCO/Lick Observatory and Department of Astronomy and Astrophysics, Univ. of \\ California, Santa Cruz, CA, 95064, E-Mail: jharris@ucolick.org, dennis@ucolick.org \\ and \\ Ian Thompson \\ Observatories of the Carnegie Institution of Washington, 813 Santa Barbara St., Pasadena, \\ CA 91101, E-Mail: ian@ociw.edu
}

${ }^{1}$ Lick Bulletin No. 1365 


\begin{abstract}
We present a detailed map of the reddening in a $1.9^{\circ} \times 1.5^{\circ}$ section of the Large Magellanic Cloud (LMC), constructed from UBVI photometry of $2069 \mathrm{O}$ and B main sequence stars. We use two reddening-free photometric parameters to determine the line-of-sight reddening to these stars. We find a mean reddening, $\langle E(B-V)\rangle_{L M C}=0.20 \mathrm{mag}$, with a non-Gaussian tail to high values. When the reddening is corrected for foreground Galactic extinction (Oestreicher et al. 1995), we find $\langle E(B-V)\rangle_{L M C}=0.13$ mag. The line-of-sight values are then interpolated onto a uniform grid with a local least-squares plane fitting routine to construct a reddening map of the region. We use the distribution of reddening values to constrain the line-of-sight geometry of stars and dust in the LMC, and to test and normalize a standard extinction correction for galaxy photometry. We attempt to distinguish between line-of-sight depth effects and structure in the dust distribution as possible causes for the observed differential reddening through this region.

We conclude: (1) that our data are consistent with a vertical exponential distribution of stars and dust in the LMC, for which the dust scale height is twice that of the OB stars; (2) that the dust distribution must be non-uniform (clumpy) to account for the full distribution of measured reddening values (i.e., line-of-sight effects alone are insufficient to explain the observed structure); and (3) that the $B$-band optical depth, $\tau_{B}$, through the observed region of the LMC is $0.69<\tau_{B}<0.82$.
\end{abstract}

\title{
1. Introduction
}

Observations of galaxy properties, and therefore of the extragalactic universe, are affected by internal dust extinction. Any correction for this extinction is complicated by the unknown relative distributions of dust and stars. Empirical efforts to determine these distributions have been based either on statistical analyses of multicolor images of large samples of galaxies, or on detailed analysis of high spatial resolution, multicolor images of a few, select nearby galaxies. Theoretical efforts have depended upon the assumption of simple geometries, even though the radiative transfer models are becoming more realistic with the inclusion of both scattering and absorption (Bruzual et al. 1988, Witt et al. 1992). Neither approach has succeeded in detail because of the complexity in interpreting integrated galaxy properties. The goal of this study is to observationally constrain the star-dust geometry in one galaxy, the Large 
Magellanic Cloud, for which we can observe lines-of-sight to individual stars. Galaxy extinction corrections depend sensitively upon several factors, including: (1) the inclination of the galaxy ( $c f$. Valentijn 1990, Burstein et al. 1991, Huizinga \& van Albada 1992, Giovanelli et al. 1994); (2) the stellar population age and metallicity gradients ( $c f$. de Jong 1996, Peletier et al. 1994); and (3) the details of the dust geometry (e.g., clumpiness; Kuchinski \& Terndrup 1996). This sensitivity is reflected by the wide range of results quoted in different studies ( $c f$. Davies \& Burstein 1995). Disney (1989), Valentijn (1990) and Burstein et al. (1991) have concluded that galactic disks are optically thick, even in their outer regions. Bosma (1992), Huizinga \& Van Albada (1992), Byun (1993), Giovanelli et al. (1994) and others have found that the optical depth is significant only near the center of disks. Further study, particularly of a galaxy in which the stars can be individually resolved, is critical to determining the importance of clumpiness and the relative distributions of the dust and different stellar populations.

The Magellanic Clouds are the most suitable galaxies for a determination of the detailed internal distribution of dust and stars in a galaxy and the subsequent effects on the observed properties. In this paper, we present a determination of the spatially-resolved reddening in a section of the Large Magellanic Cloud. Rather than relying on integrated colors or model-dependent reddening indicators, the line-of-sight reddening toward individual stars is measured using four-filter photometry. This allows us to avoid complications related to scattering and stellar population gradients.

This article is structured as follows. In \$2, we present the data and our reduction techniques. In $\$ 3$, we discuss the construction of the reddening map, including the determination of the line-of-sight reddening values, the interpolation of these values to a uniform grid, and the results from several tests of the procedure. In $\S$, we discuss the following: (1) a comparison of our reddening values to the results from previous studies of dust in the LMC, (2) our constraint on the line-of-sight geometry of stars and dust in the LMC, (3) a derivation of the total $B$-band optical depth of the LMC disk and a test of an extinction correction for the integrated photometry of spiral galaxies, (4) an investigation of whether coherent dust structure or incoherent effects, such as line-of-sight depth differences, dominate the observed differential reddening across the LMC, and (5) a positionally-dependent reddening correction applied to the photometry of all stars in this section of the LMC. We find that the distribution of dust is non-uniform, and that it differentially affects the photometry across the observed region. This result appears self-evident, but 
it is usually ignored in both empirical and theoretical treatments of internal extinction. Its inclusion represents progress toward a greater understanding of the distribution of stars and dust in the LMC.

\section{Data}

The data, reduction, and photometric calibration are described in detail elsewhere (Zaritsky, Harris, \& Thompson 1997), so we only briefly review their main characteristics here. The data come from the initial stages of a $U B V I$ photometric survey of the central $8^{\circ} \times 8^{\circ}$ of the LMC and $4^{\circ} \times 4^{\circ}$ of the SMC and were obtained using the Great Circle Drift Scanning Camera Zaritsky, Schectman, \& Bredthauer 1996) at the Las Campanas 1m Swope telescope in November 1995. The effective exposure time is set by the sidereal drift rate of a star across the field-of-view, which for this instrumental setup corresponds to about 4 minutes. The images cover a $1.9^{\circ} \times 1.5^{\circ}$ region with 0.7 arcseconds per pixel resolution. The region is centered at approximately $\alpha=5.2^{h}$ and $\delta=-67.4^{\circ}$.

The data are reduced in a fairly standard manner using DAOPHOT II (Stetson 1987) and our own algorithms that automate the process as much as possible to produce a catalog of RA, Dec, and $U, B, V$, and $I$ photometry for over one million stars. Four filter photometry is not available for every star because some stars are fainter than the respective magnitude limits in certain bands. The catalog becomes 50\% incomplete at $V \sim 21$ mag, well below the apparent magnitude of $\mathrm{OB}$ stars $(V \lesssim 17 \mathrm{mag})$. We require that a star is identified in both $B$ and $V$ to enter the catalog. Photometric uncertainties are calculated by DAOPHOT and convolved with uncertainties in the standard star calibration using Landolt (1992) standard fields to produce our final photometric uncertainties. We find that this uncertainty at worst underestimates the true uncertainty by a factor of two ( $c f$. Zaritsky, Harris, \& Thompson 1997).

\section{Constructing the Reddening Map}

The translation of stellar photometry into a reddening map involves two steps. First, we determine the line-of-sight reddening toward OB stars using two reddening-free combinations of the available photometric colors. These data will be used to constrain models of the dust geometry both along and across the line-of-sight. Second, we interpolate the line-of-sight reddening values (LOSRV) onto a rectilinear grid of points using a local least-squares plane fitting routine 
to produce a two-dimensional map of the reddening. We describe the entire procedure below.

\subsection{Selection of Program Stars}

We use only OB main sequence stars to determine the LOSRV because (1) they have small photometric errors due to their relative brightness; (2) their intrinsic colors have a negligible dependence on metallicity Oestreicher et al. 1995), so we can use Galactic stellar data to construct the relationship between reddening-free parameters and intrinsic color, despite the mean metallicity difference between the LMC and the Milky Way; and (3) the relationship between the intrinsic $B-V$ color, $(B-V)_{0}$, and the reddening-free parameter for these stars is single-valued. We select candidate $\mathrm{OB}$ stars using the photometric criteria $V<17+3.2(B-V)$ and $B-V<0.4$ (cf. Figure 11). The first criterion excludes main sequence stars later than spectral type B5 (for $(m-M)_{L M C}=18.47$; Feast \& Walker 1987). The color term in this expression anticipates the effects of reddening by placing the boundary along a line of constant spectral type, ensuring that faint stars with high reddening are not under-represented, as discussed by Oestreicher and Schmidt-Kaler (1996). The second criterion excludes Galactic main sequence stars by placing a cut blueward of the Galactic halo main sequence turn-off (Gilmore et al. 1989; cf. our Figure 1), but, unfortunately, may also exclude highly reddened $(E(B-V)>0.7$ mag $)$ LMC stars. We discuss the number of highly obscured stars excluded by this criterion in $\oint 3.3$. Finally, we exclude $\mathrm{OB}$ stars with photometric uncertainties that lie beyond the half-maxima of the uncertainty distributions $(>0.2,0.1,0.1$, and $0.1 \mathrm{mag}$ for $U, B, V$, and $I$, respectively).

As a final note about sample selection, we address the concern that slightly evolved OB stars are photometrically degenerate with reddened OB stars, and that these stars will cause us to overestimate the reddening along their lines-of-sight. Theoretical isochrones (Bertelli et al. 1994) show that as an OB star evolves redward in $B-V$, it becomes redder in $U-B$ in such a way that it remains indistinguishable from an unreddened upper main sequence star. The supergiant and main sequence loci diverge only redward of $B-V \sim 0$, a situation that will require us to eliminate the more evolved supergiant stars from our sample ( $c f . \$ 3.2)$. 


\subsection{Determining the Line-of-Sight Reddening Values}

We utilize two reddening-free photometric parameters to measure the LOSRV,

$$
\begin{gathered}
Q_{1}=(U-B)-0.76(B-V)-0.05(B-V)^{2} \\
Q_{2}=(U-B)-0.60(V-I)+X(V-I)^{2} .
\end{gathered}
$$

These are equivalent to a rotation of the two-color diagram, so that the reddening line is parallel to one axis. $Q$ measures the distance along the axis orthogonal to the reddening line; thus $Q$ is reddening-free. The quadratic terms correct for slight variations in the slope of the reddening line for different spectral types. $Q_{1}$ is a derivative of the familiar Johnson and Morgan (1953) parameter for the $U B V$ photometric system. The coefficient of the linear $(B-V)$ term is the ratio of color excesses $E(U-B) / E(B-V)$ evaluated from the average LMC extinction curve outside 30 Dor (Eitzpatrick 1985). The coefficient of the quadratic term is drawn directly from Galactic studies (Hiltner \& Johnson 1956). $Q_{2}$ is a hybrid of the Johnson and Morgan $Q$ and the $Q$ presented by Grieve and Madore (1986) for the BVI system. The coefficient of the linear $(V-I)$ term is again the ratio of color excesses $E(U-B) / E(V-I)$ evaluated from the average extinction curve for the LMC outside 30 Dor. To determine $X$ empirically, we construct an artificial stellar sample, adopt a distribution of reddening values consistent with our initial reddening estimates using only $Q_{1}$ and the LMC extinction curve, and add random reddening errors with $\sigma=0.04$ mag, consistent with the data. We find $X$ to be 0.08 by requiring $\left\langle Q_{2}\right\rangle=\left\langle Q_{1}\right\rangle$. In practice, our final results are nearly independent of $X$ because a star at the extreme red end of our color range has a quadratic term in $Q_{1}$ of 0.008 , corresponding to a correction to $E(B-V)$ of only 0.002 magnitudes. A similar argument demonstrates that adopting the Galactic value for the coefficient of the quadratic term in $Q_{1}$ is acceptable. The quadratic terms only become important for highly obscured stars, which we have excluded from the current sample.

The line-of-sight color excess, or reddening, is measured from a comparison of the observed color (e.g., $B-V$ ) of a star with a particular $Q$ value, and the intrinsic color (e.g., $\left.(B-V)_{0}\right)$, of an unreddened star with the same $Q$ value (cf. Figure 2). The intrinsic sequences for OB stars in Figure 2 are determined by fitting a line in each $Q$-color diagram to a compilation of observations of unreddened Galactic OB stars (Straižys 1992). The rms dispersions of the Straižys data about the best-fit lines are $0.006 \mathrm{mag}$ and $0.004 \mathrm{mag}$ for $Q_{1}$ and $Q_{2}$, respectively. The offset of the vast majority of stars in our sample 
toward redder colors indicates that most of these stars are reddened. The correspondence between the LOSRV derived from the two $Q$ 's is illustrated in Figure 3. We have removed stars from the sample if their reddening values as derived from $Q_{1}$ and $Q_{2}$ differ at more than the $5 \sigma$ level, or if $E(B-V)<-5 \sigma$, where $\sigma=0.039 \mathrm{mag}$, the mean propagated LOSRV error. If a star is rejected by either of these conditions, it suggests either that the star is unusual or that there is a problem with the data for that star. Twenty-seven stars (out of 2619) were rejected for this reason.

The standard deviation of the remaining stars from the line $E(B-V)_{Q_{2}}=E(B-V)_{Q_{1}}$ is 0.044 mag, only slightly larger than the mean propagated LOSRV error $(\sigma=0.039 \mathrm{mag})$. The small additional scatter introduced by the two different calculations of $E(B-V)$ demonstrates that our choice of coefficients for $Q_{1}$ and $Q_{2}$ is reasonable and that the extinction curve in the observed region of the LMC is not grossly different from the adopted curve. We adopt the mean of the two color excesses, weighted inversely by their uncertainty, as the LOSRV in our subsequent analysis and refer to this quantity as $E(B-V)$. Because $Q_{1}$ and $Q_{2}$ are not entirely independent, we adopt the smaller of the propagated uncertainties of $E(B-V)_{Q_{1}}$ and $E(B-V)_{Q_{2}}$, rather than the uncertainty of the mean, for the uncertainty in $E(B-V)$.

Finally, we de-redden the OB stellar photometry using the LOSRV and note that most stars collapse tightly to the intrinsic main sequence ( $c f$. Figure 1), but that a surprisingly large fraction $(\sim 20 \%)$ collapse instead to $B-V=0$

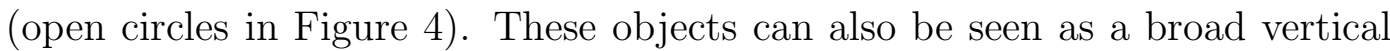
band in Figure 2 at $Q \sim 0$. Their coherence in Figure 2, their uniform spatial distribution, their spread in magnitudes, and the consistent reddening determinations from $Q_{1}$ and $Q_{2}$ all suggest that these objects are not the result of faulty data or reduction problems. Examination of these stars in the two-color diagram shows that they are probably supergiant stars in the LMC. While these supergiants are degenerate with reddened OB stars in the color-magnitude diagram (CMD), the degeneracy is broken in the two-color diagram. The supergiants lie along a sequence in the two-color diagram that is nearly parallel to the reddening line, causing the de-reddening algorithm to collapse all of these stars to the same $B-V$ color. This behavior allows us to easily exclude these stars from our sample, by keeping only those stars with $B-V<-0.1$ mag after de-reddening $\left((B-V)_{0}=-0.17\right.$ mag for spectral type B5, the latest type allowed by our faint magnitude limit). After this final cut, we are left with 2069 OB main sequence stars with measured LOSRV in this section of the LMC (from an initial sample of 2619 candidate OB stars). 
We test the validity of our estimated uncertainties by examining the distribution of the LOSRV ( $c f$. Figure 5). Had we severely underestimated the errors, a larger than expected number of stars would have scattered toward unphysical, negative inferred reddening values. The stars with $E(B-V)<0$ are well-fit by the left half of a Gaussian centered at zero, with a FWHM characterized by the propagated uncertainties $(\langle\sigma\rangle=0.039 \mathrm{mag})$, as shown by the solid curve in Figure 5. Therefore, they are consistent with a small, zero-reddening population plus photometric errors.

Finally, because we are interested in the internal distribution of dust in the LMC, we need to correct the reddening values for foreground Galactic extinction. We use the foreground reddening map for the LMC of Oestreicher et al. (1995). This foreground map has 10 arcmin pixels and is based on the photometry of 1409 Galactic stars in the direction of the LMC. Figure 5 also shows the foreground-corrected histogram. The inclusion of the Oestreicher et al. uncertainties into our error budget increases the mean propagated LOSRV error to $\langle\sigma\rangle=0.045 \mathrm{mag}$. Note in Figure 5 that the stars with $E(B-V)<0$

are still fairly well-fit by a $\sigma=0.045$ Gaussian centered at zero, again consistent with the presence of a zero-reddening population plus photometric errors. The amplitude of this Gaussian is larger, simply because there are now more stars with $E(B-V)<0$ than there were before the foreground extinction correction.

\subsection{Highly Obscured Stars}

Our photometric selection criteria bias our sample against highly obscured stars. We originally excluded stars with $B-V>0.4 \mathrm{mag}$, because these are photometrically degenerate with a population of Galactic main sequence stars, and so we cannot detect $\mathrm{OB}$ main sequence stars with $E(B-V) \gtrsim 0.7 \mathrm{mag}$.

To determine whether there is a significant population of such stars, we perform a "pseudo-dereddening" of the photometry of red giant branch stars that is similar to blue envelope techniques used to determine the reddening of LMC supergiants. We cannot perform a true reddening analysis of these stars, because the intrinsic colors of these red giant stars are not independently known; the blue envelope is assumed to be the reddening zeropoint. However, the assumption of a small mean extinction is not likely to be wrong by more than 0.1 or 0.2 magnitudes, because the position of the red clump is approximately where it is expected, for $(m-M)_{L M C}=18.5 \mathrm{mag}$. We isolate a region of the CMD bounded by two reddening lines, and by a line selected to follow the blue envelope of the red giant branch. This region is shown in Figure 11. We exclude 
stars with large photometric errors ( $>0.2 \mathrm{mag}$ for $U ;>0.1 \mathrm{mag}$ for $B, V, I$ ) from this analysis. We then slide each star in this region along a reddening vector in the $B-V, V$ CMD until it lies on the blue envelope of the red giant branch. Figure 6 shows a histogram of the resulting reddening values. We find very few highly obscured $(E(B-V)>0.7 \mathrm{mag})$ red giant branch stars, even though stars reddened by as much as $E(B-V)=1.2 \mathrm{mag}$ are above our detection limit. We conclude that there are no large areas of high obscuration in this region of the LMC, unless they are extremely obscured $(E(B-V)>1.2$ mag).

Before discussing the geometric implications of the LOSRV distribution, we finish our discussion of the procedure by presenting the construction of a two-dimensional reddening map for this region of the LMC.

\subsection{Interpolating the LOSRV}

The mean projected density of OB stars in this region of the LMC, roughly $0.25 \mathrm{OB}$ stars per square arcmin, enables us to construct a map with an average resolution of $\sim 10$ arcmin. The scientific value of such a map depends on whether the observed LOSRV are spatially correlated, and are therefore sampling coherent dust structure in the LMC. Incoherent effects may dominate, making the map meaningless. One such effect arises because we have collapsed a three-dimensional distribution of dust into a two-dimensional map. The different LOSRV may simply indicate the relative depth of the OB stars along the line-of-sight within a uniform dust layer. Another incoherent effect may arise if there are large-amplitude reddening variations on small scales across the plane of the sky. Such variations would be undersampled by our algorithm, leading to the detection of false structure ( $c f$. 83.5$)$.

Even with these potential difficulties, the construction and analysis of the map will help determine the degree to which these issues affect our final inferences regarding the reddening across the LMC. In $\$ 4.4$, we conclude that the map contains valuable information about the dust distribution in the LMC, even though it is somewhat affected by both line-of-sight depth effects and unresolved small-scale variations.

The initial step in constructing the map is the selection of an appropriate grid resolution, which is complicated by the non-uniform distribution of OB stars ( $c f$. Figure 7). To take advantage of the resolution provided by localized high concentrations of $\mathrm{OB}$ stars, we map the reddening of the OB stars onto a grid with spacings of 1.2 arcmin in right ascension and declination. However, 
to avoid being dominated by noise in the region where OB stars are scarce, we adopt an interpolation method that incorporates an adaptive effective resolution scale. This approach reduces the correlation between the map uncertainty and the local surface density of OB stars.

We use the following algorithm to generate the reddening map. We search for OB stars with measured LOSRV within a circular area of sky of radius 1.2 arcmin centered on each gridpoint. If we find 20 or more LOSRV within the search area, we perform a least-squares plane fit to the reddening values. If we find fewer than 20, then the search radius is increased by 1.2 arcmin, and we repeat the search. We continue increasing the search radius until we find at least 20 LOSRV. When 20 values are found, a plane is fit and all grid points within the final search area are assigned the value of the best-fit plane at their location. Because search areas overlap, each gridpoint will be assigned multiple interpolated reddening values. We adopt the weighted mean of these values for the reddening at that grid point, and use the dispersion of the values about the mean as an estimate of the reddening map error. The resulting reddening map is presented in Figure 8a. A map of the dispersion of the assigned interpolated reddening values of each gridpoint is shown in Figure 8b.

Figure 8 includes foreground extinction; it should be used to correct LMC photometry. However, to examine the internal structure of dust in the LMC, it is necessary to use the foreground-corrected LOSRV ( $c f$. Figure 5). The corrected reddening map is shown in Figure $9 \mathrm{a}$, and the map of the dispersion is shown in Figure 9b.

\subsection{Tests of the Mapping Procedure}

The variation of the LOSRV along different lines of sight far exceeds what is expected from the observational uncertainties. We discuss the nature of the structure visible in Figures 8 and 9 in $\$ 4.4$. For now we simply test whether our interpolated map is an accurate representation of the variations in the LOSRV. We do so by attempting to recover artificial reddening maps with our least-squares plane fitting algorithm. We start by taking the coordinates of our 2069 OB stars and assigning to them LOSRV drawn from two-dimensional sinusoidal surfaces that represent the artificial maps. Each simulated LOSRV is assigned a random photometric error consistent with the observational uncertainties. We vary the amplitude and spatial frequency of the surfaces to derive limits on our ability to detect and resolve reddening variations. Some representative reddening maps and the corresponding recovery by our 
interpolation routine are shown in Figure 10.

First, we examine our sensitivity to variation on different spatial scales. For these tests, the amplitude of the reddening variations is held constant at $E(B-V)_{\max }=0.7$, consistent with the variations in the observed LOSRV. In relatively dense regions, such as in an $\mathrm{OB}$ association, we can trace variations on scales $\gtrsim 7$ arcmin, while in the sparsest regions we can only trace variations on scales $\gtrsim 15$ arcmin. Even when the local variations are unresolved, the mean reddening value is recovered, except for the false structure due to undersampling in the very high frequency map ( $c f$. $\$ 4.4 .2)$. If the actual reddening in low stellar density regions varies with as much amplitude $(0.7 \mathrm{mag})$ on such small scales ( $\lesssim 7$ arcmin), then our map may contain false structure.

Next, we examine our sensitivity to different amplitudes. The wavelength of the variations is held fixed at approximately 15 arcmin, and we examine the recovery of amplitudes set to $8 \sigma, 4 \sigma, 2 \sigma$ and $1 \sigma$. In each case, the surfaces are normalized to the same mean reddening value, and the greyscale in Figure 10 is held constant. We find that the technique is sensitive to $>2 \sigma$ reddening amplitudes, where $\sigma$ is the propagated LOSRV error. Even when these low amplitude variations are not recovered, the mean value of the reddening in that region is recovered.

Our final test of the interpolation technique, a self-consistency check, is the comparison of the LOSRV of OB main sequence stars to the reddening values estimated from the map at the position of the OB stars ( $c f$. Figure 11). For stars with $E(B-V)_{Q} \lesssim 0.2$, the two determinations correlate, with a zeropoint offset of $0.021 \mathrm{mag}$. The rms scatter of these low-reddening stars about the best fit line (the dotted line in Figure 11) is 0.056 , which is consistent with the characteristic error of the reddening map values $(\sigma=0.06)$. Above $E(B-V)=0.2$, the interpolation systematically underestimates the LOSRV because the algorithm smooths over sharp peaks in the LOSRV distribution. This smoothing effect is less of a problem in high density, high LOSRV regions. For example, the peak in the reddening map (Figure 8 $8 \mathrm{a})$ near $\left(5.25^{h},-67.4^{\circ}\right.$ ) corresponds to a dense OB association. Most of the high LOSRV stars lie outside of OB associations and are surrounded by stars with lower LOSRV, which may indicate highly localized reddening (e.g., circumstellar envelopes). If these high LOSRV are truly localized, the map is a more accurate representation of the general reddening distribution than one might infer from Figure 11 . 


\section{Discussion}

We will use the distribution of LOSRV and the reddening map to perform several investigations of the dust distribution in the LMC. First, we compare our LOSRV distribution to results from previous studies of reddening in the LMC (§4.1). Second, we use our data to place constraints on the line-of-sight geometry of stars and dust in the LMC (\$4.2). Third, we test an analytic technique used to correct the luminosity of distant galaxies for internal extinction (\$4.3). Fourth, we investigate the nature of the observed LOSRV variations and subsequent map structure (\$4.4). We examine the effects of line-of-sight depth differences and small-scale dust structure, the correlation of the LOSRV with OB star density, and the spatial coherence of the LOSRV. We conclude that a positionally-dependent reddening correction does provide some improvement to the single-value reddening correction and that it can highlight regions of potentially high extinction, as well as regions of favorable low extinction. We have not, and cannot, fully account for the line-of-sight depth effects, the undersampling effects, or the possible bias introduced by sampling the ISM with massive stars. Nevertheless, the LOSRV and the reddening map provide the most detailed information on the distribution of dust in this region of the LMC yet available. Finally, we apply a positionally-dependent reddening correction to the photometry of this region $(\$ 4.5)$.

\subsection{Comparison to Previous Studies}

Previous studies have typically found that the mean reddening toward LMC stars is $\lesssim 0.1 \mathrm{mag}$ ( $c f$. Feast et al. 1960, Isserstedt 1975, Grieve \& Madore 1986). More recently, Hill et al. (1994) found $\langle E(B-V)\rangle=0.15 \mathrm{mag}$, Massey et al. (1995) found $\langle E(B-V)\rangle=0.13 \mathrm{mag}$ and Oestreicher and Schmidt-Kaler (1996) found $\langle E(B-V)\rangle=0.16 \mathrm{mag}$, although Hill et al. focused on OB associations, rather than the entirety of the LMC. It should also be noted that the Hill et al. and Massey et al. values are not corrected for foreground extinction, while the Oestreicher and Schmidt-Kaler value is corrected. Oestreicher and

Schmidt-Kaler argue that other studies underestimate the mean reddening either because they use Galactic intrinsic stellar colors for supergiants, or because the faintest stars are biased toward low reddening values because of a color-independent magnitude limit. The former causes a bias because supergiants in the LMC are intrinsically bluer than their Galactic counterparts due to their lower metallicity (Oestreicher \& Schmidt-Kaler 1996). The latter causes a bias because highly reddened, faint stars are preferentially lost below 
the faint magnitude limit. We avoid the supergiant color issue by only using main sequence OB stars, whose intrinsic colors are insensitive to metallicity changes (Oestreicher et al. 1995). We address the completeness issue by introducing a color-dependent limiting magnitude cut that is not biased against faint, reddened stars relative to faint, unreddened stars. We are able to construct this color-dependent cut because the faintest OB stars are many magnitudes brighter than our limiting magnitude. Our analysis (corrected for foreground Galactic extinction) yields $\langle E(B-V)\rangle=0.13$ mag; consistent with the most recent results. However, our mean value is based on only $\sim 4$ percent of our eventual total LMC survey area, and this area may be systematically different from the majority of the LMC.

In addition to presenting the mean value, two of the recent studies have included histograms of the LOSRV. The position and width of our low-reddening peak is consistent with the LOSRV distributions observed by Oestreicher and Schmidt-Kaler and Massey et al. However, Massey et al. do not find a significant high-reddening tail. This may be partly due to their smaller sample size, but when we renormalize our histogram to match their number of OB stars, we still find a more significant tail in our data. We suspect that the discrepancy exists because the Massey et al. sample does not employ a color-dependent faint magnitude limit; thus, it is biased against the detection of highly reddened stars near the faint magnitude limit. Furthermore, they require spectroscopy to determine the intrinsic colors, so their limiting magnitude is quite bright (roughly $V=15 \mathrm{mag}$ ). When we limit our sample with a color-independent magnitude limit at $V=15 \mathrm{mag}$, we obtain a similar histogram to that of Massey et al.

\subsection{Dust Geometry Models}

The LOSRV are a measurement of the total column density of dust toward the OB stars. The LOSRV depend both on the physical density of the ISM, and the line-of-sight depth of the star inside the dust layer. In this section, we discuss our constraints on the relative distribution of stars and dust along the line-of-sight in the LMC by comparing the observed LOSRV distribution (Figure 5) to those produced by models of various star and dust distributions. We begin with simple models in which the stars are uniformly distributed in a slab of finite thickness, and the dust is either in a slab that lies in front of the stars, uniformly mixed with the stars, or in an infinitesimally thin midplane sheet. We also examine the more realistic situation of exponential disk distributions of stars 
and dust. We allow the scale height of the stars and dust to vary independently in these models, and present three representative cases here: $h_{s} / h_{d}=0.5,1.0$, and 2.0, where $h_{s}$ is the scale height of stars and $h_{d}$ is the scale height of dust. In each of these six models, we generate the intrinsic stellar photometry from a theoretical isochrone (Bertelli et al. 1994; age=4 Myr (their youngest)), add photometric errors, place the star within the geometrical model, and redden its colors in proportion to the amount of foreground dust according to the LMC extinction curve. We define the mean optical depth in each model to match the observed mean $(E(B-V)=0.13 \mathrm{mag})$. The simulated LOSRV distributions are shown in Figure 12.

The slab models produce LOSRV distributions markedly different from that observed. Aside from the unphysical premise of foreground sheet model, the resulting LOSRV distribution is much narrower than the observed distribution. This result is non-trivial because standard mean-value reddening corrections to LMC photometry imply this geometry. The midplane sheet model generates a wider LOSRV distribution, but the distribution is strongly bimodal - half of the stars are reddened a non-zero constant amount, and the other half are unreddened. Of the three slab models, the mixed model produces the best match to the observations, but the asymmetric tail to high LOSRV is still not reproduced and the peak in the distribution is too flat. A more complex model is needed.

Because the LMC is a disk galaxy ( $c f$. Prévot et al. 1989 and references therein), we expect the stars and dust to be well approximated by a vertical exponential distribution (Blaauw \& Schmidt 1965, de Grijs \& van den Kruit 1996). We model the distribution of stars and dust along the line-of-sight as exponentials about a fiducial midplane that is perpendicular to the line-of-sight. The inclination of the LMC is between $\sim 33^{\circ}$ and $45^{\circ}$ (Westerlund 1990), but the inclination only introduces a constant factor of $\sin (i)$ to both the star and dust scale heights. Since the ratio of the scale heights of the stellar and dust distributions is the only free parameter of the model, the inclination does not affect our analysis. As $h_{s} / h_{d} \rightarrow 0$, the model reduces to the foreground sheet model (since all the stars are equally reddened); as $h_{s} / h_{d} \rightarrow \infty$, it reduces to the thin midplane layer model; and intermediate models resemble the mixed model. As one increases $h_{s}$ relative to $h_{d}$ the reddening distribution will widen, but it will also become increasingly bimodal. Again, we set the mean of the model LOSRV distribution to match the data $(\langle E(B-V)\rangle=0.13)$. Finally, we stress that $h_{s}$ refers to the scale height of OB stars, not the general population of LMC stars. 
Many dust models invoke a plane-parallel geometry ( $c f$. Bruzual et al. 1988, Di Bartolomeo et al. 1995) or an exponential disk geometry ( $c f$. Disney et al. 1989, Byun et al. 1994, Corradi et al. 1996). However, one geometrical aspect that poses serious difficulties to both empirical and theoretical treatments of this problem, and has therefore generally been ignored, is clumpiness in the distribution of stars and/or dust (for exceptions, see Boisse 1990, Hobson \& Scheuer 1993). As illustrated in Figure 12, the model with $h_{s} / h_{d}=0.5$ best matches the width of the observed distribution peak. This ratio is consistent with the vertical structure of most spiral galaxies. Allen (1973) found that the typical OB stars-to-dust scale height ratio is 0.42 . However, this smooth exponential model fails to account for both the tail of highly reddened stars and the number of stars with $E(B-V)<0$ mag. The simplest modification of the model is to invoke a clumpy component to the distribution of dust.

To simulate a clumpy dust distribution, we randomly select a fraction of the lines-of-sight to be more highly reddened than the exponential dust envelope would predict. These lines of sight are given LOSRV drawn from either (a) an exponential or (b) an offset Gaussian reddening distribution. We also add "holes" to the distribution to match the data with $E(B-V)<0$. The holes are modeled simply by randomly selecting a fraction of the lines-of-sight to have zero reddening. These clumpy-model parameters are interactively adjusted until the models match the data. In the exponential model, $40 \%$ of the stars are assigned the midplane reddening, plus an additional reddening drawn randomly from an exponential distribution with a reddening scale length of 0.17 mag. In the Gaussian model, $50 \%$ of the stars have reddening drawn from a Gaussian with a mean value of $0.2 \mathrm{mag}$ and $\sigma=0.2 \mathrm{mag}$ (the model excludes Gaussian reddening values below zero). In both cases, we set $5 \%$ of the lines-of-sight to have zero reddening. There is yet no physical motivation for these particular model parameters. The requirement of a clumpy component suggests that at least some of the structure in the reddening map is real, and that approximately half of the OB stars have reddening values that cannot be explained by a smooth exponential disk model.

\subsection{Internal Extinction in Spiral Galaxies}

The LOSRV and the geometric models of dust and star distributions allow us to derive the total optical depth through the LMC and to compare the extinction as derived on a star-by-star basis to that derived from global properties. This provides an independent check on whether the integrated magnitudes of more 
distant galaxies are being properly corrected for internal extinction.

We now derive a quantitative measurement of the total $B$-band optical depth for the LMC, which we present as new evidence for the ongoing debate over the optical thickness of disk galaxies (see $\S 1$ ). We begin with our midplane reddening measurement, $E(B-V)=0.13$ mag. By applying the standard Galactic optical extinction curve (fine for the LMC (Fitzpatrick 1985)), this reddening is equivalent to a $B$-band extinction, $A_{B}$, of 0.533 mag. This extinction is directly transformed to the optical depth using $\tau_{B}=\frac{A_{B}}{1.086}=0.49$. The total optical depth of the observed region of the LMC disk is assumed to be twice this midplane value. The face-on optical depth is given by $\tau_{B}^{0}=0.98 \times \cos (i)$. The inclination of the LMC is between $33^{\circ}$ and $45^{\circ}$, therefore the face-on optical depth of the LMC disk is between $\tau_{B}^{0}=0.82$ and $\tau_{B}^{0}=0.69$.

This optical depth measurement can be compared to an extinction correction for spiral disks provided by Tully and Fouqué (1985, hereafter TF). There may be concerns about applying such a correction to an irregular galaxy like the LMC; however TF themselves include such galaxies in their sample. Furthermore, the only property required of a galaxy for this model to work is that it be a disk system. The extinction correction is based on a finite, plane-parallel midplane absorbing layer, and it is given by:

$$
A_{B}=-2.5 \log \left[f\left(1+e^{-\tau_{B} \sec (i)}\right)+(1-2 f)\left(\frac{1-e^{-\tau_{B} \sec (i)}}{\tau_{B} \sec (i)}\right)\right],
$$

where $\tau_{B}$ is the face-on optical depth of the absorbing layer, and $(1-2 f)$ is the fractional thickness of this layer, compared to the thickness of the stellar slab (for simplicity, stars of all types have the same vertical distribution). TF adopt $\tau_{B}=0.55$ and $f=0.25$ as the best-fit values to their sample of 600 nearby spiral galaxies, independent of galaxy type. These parameters are poorly constrained. According to their Figure 5, values of $\tau_{B}$ between 0.4 and 1.2, and values of $f$ between 0.0 and 0.4 are acceptable. In principle, this equation could be used to derive a galaxy's true, extinction-free luminosity. In practice, because of the poor constraint on $\tau_{B}$, it is used only to correct a galaxy's photometry to its face-on values. The correction from face-on magnitudes to dust-free magnitudes has remained largely unknown.

While our optical depth measurement is higher than TF's prediction, it is well within their acceptable range. A straightforward application of the mean TF correction to the integrated photometry of the LMC would result in a $16 \%$ underestimate of the $B$-band luminosity of the LMC, compared with a correction based on our B-band extinction. Recently, Tully et al. (1997) 
have refined their model parameters to values of $\tau_{B}=0.8$ and $f=0.1$, and

find a dependence of these values on galaxy type. Their new calibration would suggest that a galaxy like the LMC should have $\tau_{B} \sim 0.4$, resulting in a $22 \%$ underestimate of the $B$ luminosity. We conclude that (1) the optical depth through the LMC is relatively high $\left(\tau_{B} \sim 0.75\right)$ even at a radius of $2 \mathrm{kpc}$ and disregarding the tail of high LOSRV (this is independent of the validity of the TF model), (2) that if SBm galaxies do indeed have lower optical depths than larger spirals (as suggested by Tully et al. 1997), then the latter likely have $\tau_{B} \sim 1$, and $(3)$ that the statistical reddening correction presented by $\mathrm{TF}$ is valid, at least for this region of the LMC, to $\sim 20 \%$.

These results come with two important caveats. First, our optical depth measurement is based on the reddening of OB stars. This population may be in regions of higher than average extinction, in which case we have overestimated the mean optical depth. Second, investigators generally use a correction like that in TF to correct galaxies to face-on magnitudes, rather than to dust-free magnitudes, and relative reddening differences may be more precise than the $20 \%$ uncertainty found above.

\subsection{The Nature of Observed Reddening Variations}

To determine whether the reddening map is useful for de-reddening observations within this region, we need to establish whether incoherent effects, such as different line-of-sight depths of the OB stars and small-scale dust density variations, dominate the observed structure. Several lines of argument will lead to the same conclusion: incoherent effects of line-of-sight depth differences and small-scale reddening variations are present, but they do not dominate the observed structure in the reddening map.

\subsubsection{Line-Of-Sight Depth Effects}

First, we must determine how much of the structure seen in the reddening map is due to line-of-sight depth differences among the OB stars, rather than to density variations in the dust across the line-of-sight. If the observed structure is dominated by line-of-sight-depth effects, we would expect the following: (1) the line-of-sight models constructed in $\$ 4.2$ should reproduce the observed LOSRV distribution without a need to invoke clumpiness; (2) OB stars in associations should be similarly reddened, while field OB stars should have higher LOSRV dispersion; (3) the LOSRV of each OB star should indicate its relative depth 
along the line-of-sight; and (4) random re-assignment of the LOSRV should not affect the spatial correlation of the LOSRV. We have already demonstrated (\$4.2) that line-of-sight depth differences in a smooth dust envelope cannot reproduce the observed LOSRV histogram. We address (2) and (3) next, and (4) in $\$ 4.4 .2$.

OB stars in associations will be similarly reddened if line-of-sight depth effects dominate the LOSRV, since the OB stars in an association are localized along the line-of-sight. Field OB stars are, in general, distributed widely along the line-of-sight. They will therefore have larger LOSRV dispersion. The two obvious $\mathrm{OB}$ associations in this area of the LMC have differential reddening that is at least as large as that among the field OB stars ( $c f$. Figure 13).

If depth effects dominate the LOSRV, then Figure 13 can be interpreted as a plot of the spatial distribution of OB stars in the LMC along the line-of-sight. The unphysical, apparently larger, extent of the far side of the LMC implied by this Figure is an indication that, at least for the high LOSRV, line-of-sight

depth differences cannot fully explain the observed reddening, even for the field OB stars.

\subsubsection{The Reddening Coherence Length}

As a final test of the clumpiness in the dust distribution, we measure the coherence length of the LOSRV. Small scale variations in the dust distribution can produce false structure in our reddening map ( $c f$. Figure 10). The LOSRV will not be spatially coherent if depth differences or these small-scale variations dominate. We attempt to measure a reddening coherence length by calculating a correlation function for the LOSRV in the following manner. Beginning with the LOSRV for one star, $L_{1}$, we calculate $\left|L_{1}-L_{i}\right|$, where $L_{i}$ represents the reddening for the $i$ th star. We do this for every star, considering each pair once. The pairs are binned by their angular separation, and the median reddening difference among the pairs in each bin is taken as characteristic for that separation. This procedure is carried out for four populations of OB stars: all OB stars, field OB stars only, low-reddening OB stars, and low-reddening field OB stars. These populations allow us to determine if the coherent structure is restricted to high reddening values, or to areas of high stellar density (OB associations). We also randomly re-assign the LOSRV in each of these four populations and calculate the median LOSRV difference at each radius. The correlation is then characterized by the ratio of the median difference in the original data to that in the randomized data. The results are shown in Figure 
14.

The decline in the normalized absolute difference as $r \rightarrow 0$ indicates that the LOSRV are correlated at small separations. The lack of a perfect correlation as $r \rightarrow 0$ (i.e. the normalized difference does not go to zero) indicates that either line-of-sight depth effects, small-scale variations, or both are present in the dust distribution. Figure 14 also demonstrates that there is little or no coherence beyond $\sim 20$ arcmin (the planes used to construct our reddening map are always fit over a region smaller than 20 arcmin), suggesting that maps with resolution $\gtrsim 20$ arcmin contain little spatial reddening information. The anti-correlation beyond one degree of separation may indicate a global reddening gradient in

this field. Again, we conclude that the observed structure in the reddening map in part represents true structure in the dust distribution. All four populations of OB stars exhibit similar coherence properties, indicating that coherent reddening structure can be inferred, even among the field population of $\mathrm{OB}$ stars, and even when the LOSRV are rather low $(E(B-V)<0.2 \mathrm{mag})$.

\subsubsection{Correlation of the Reddening with OB Star Density}

Because the dust distribution is somewhat clumped, one might expect that areas with a locally high star formation rate, which correlate spatially with dense areas of the ISM (Schmidt 1959), might lie in regions of higher than average dust density. We find that there is no significant correlation between LOSRV and projected OB star density, except possibly at low stellar densities. Likewise, previous studies of reddening in OB associations (e.g., Hill et al. 1994) have not found higher average reddening than studies of field stars (e.g., Oestreicher \& Schmidt-Kaler 1996, Massey et al. 1995). A comparison of Figures 0 and 8 reveals that although regions of high OB star density often have relatively low reddening, they tend to have nearby regions of high reddening. This may be an indication of the early stages of the disruption of the ISM caused by the high-energy radiation of OB stars. In particular, if we construct a high-resolution reddening map for the region surrounding the largest OB association (near $\alpha=5.23^{h}, \delta=-67.4^{\circ}$ ), we see this effect in detail. We improved the resolution by requiring six stars instead of twenty for each plane fit, and increasing the density of gridpoints by a factor of four. This map is shown in Figure 15, and the approximate position of the center of the OB association is marked. It shows a shell-like structure of high reddening just south of the center of the OB association, with relatively low reddening at the association's center. 


\subsection{The Reddening Correction for Non-OB Stars}

Since the reddening map is, at least in part, tracing coherent dust structure in the LMC, we use the map to de-redden all 1.1 million stars in this section of the LMC. To estimate the reddening of each star, we perform a bi-linear interpolation of the four nearest map pixel values. As a test of how well our positionally-dependent correction worked, we reconstruct the $B V$ CMD and examine the width of the de-reddened stellar sequences. We find that the sequences are generally about the same width as before; the upper main sequence has tightened up, but the red giant branch has gotten slightly wider. This indicates that the correction estimates have not been dominated by line-of-sight depth effects or small-scale variations, but also that the map is not a full description of the dust distribution. In particular, the reddening for older stars, e.g. red giants, appears to be slightly different than that of the OB stars. This may be an indication of the migration of these older stars away from the dense regions of the ISM.

\section{Summary}

Based on the $U B V I$ photometry of main sequence OB stars, we have measured the line-of-sight reddening along 2069 lines-of-sight in the LMC. The distribution of these reddening values reveals that there is significant differential reddening in the LMC. We find that this can be partially explained by the different line-of-sight depths of the OB stars inside a smooth envelope of dust, but that this explanation falls short of fully accounting for the shape and width of the reddening distribution. We conclude that there is some structure in the distribution of the dust. Although this may seem trivial given our knowledge of dust in our Galaxy, all current reddening corrections and most models of external galaxies adopt a smooth dust distribution. The LMC provides an opportunity to test these models.

We constructed line-of-sight geometric models to constrain the relative distributions of stars and dust perpendicular to the LMC midplane. We found that the best model has exponentially distributed stars and dust, with the dust scale height twice that of the OB stars. We also found that the tail to high line-of-sight reddening values (LOSRV) could not be reproduced by any simple line-of-sight geometry. The optical depth through this region of the LMC is $0.69<\tau_{B}<0.82$, for an inclination angle $33^{\circ}<i<45^{\circ}$.

We used our measurement of optical depth of the LMC to investigate how well a straightforward application of a semi-empirical extinction correction like 
that provided by Tully and Fouqué 1985 (TF) recovers the dust-free luminosity of this galaxy. We found that the LMC contains more obscuring material than is predicted by this correction, although the optical depth estimates of TF are quite uncertain and the optical depth inferred from OB stars may be an overestimate.

We examined whether the structure in the reddening map is due to coherent reddening variations across the LMC's projected surface, or to line-of-sight depth effects and unresolved small-scale variations in the dust density. We found that the latter effects are not entirely responsible for the observed structure for four reasons. First, in a smooth-envelope model where the observed stucture is due to line-of-sight depth differences, stars in associations should be more uniformly reddened than stars in the field. We did not observe this effect. Second, if the reddening is proportional to the star's position along the line-of-sight within the dust envelope, then the reddening can be used to infer each star's depth in the LMC. However, the inferred line-of-sight distribution has unphysical structure. Third, our dust-star geometric models are only partially successful in reproducing the observed LOSRV distribution. In particular, fitting the tail of high LOSRV required a clumpy component of dust. Fourth, we found that the LOSRV are partially coherent for angular separations $\lesssim 20$ arcminutes. This result indicates that the observed reddening structure is not dominated by either line-of sight effects or unresolved variations, both of which should produce incoherent LOSRV, at least among the field OB stars.

This work comes in a long line of studies of reddening in the Magellanic Clouds based on observation of the most luminous member stars. We have been able to extend those studies by examining the distribution of dust at high spatial resolution over a large region. It is evident that simple models for the distribution of dust fail to match the observations. There is, therefore, the potential for misinterpretation of the integrated photometry of more distant galaxies. An understanding of the rich and complex structure of dust in the Clouds is complicated by the unknown relative distributions of stars and dust along the line-of-sight. The combination of our full survey (which will have approximately 20 times as much data as presented here), infrared observations from ISO and IRAS, and HST observations of galaxies seen through the Clouds should enable further progress.

Acknowledgments: We gratefully acknowledge financial support from a NASA 
LTSA grant (NAG-5-3501) and an NSF grant (AST-9619576), support for the construction of the GCC from the Dudley Observatory through a Fullam award and a seed grant from the Univ. of California for support during the inception of this project. We also thank the Carnegie Institution for providing telescope access, shop time, and other support for this project, and the staff of the Las Campanas observatory, in particular Oscar Duhalde, for their usual excellent assistance. Finally, we thank M. O. Oestreicher for generously providing us with his foreground reddening map data, R. Brent Tully for an extremely useful conversation on the opacity of spiral disks, Katherine $\mathrm{Wu}$ for a very helpful reading of an earlier draft of this work, and the anonymous referee for careful and valuable comments. 


\section{References}

\section{REFERENCES}

Allen, C. W., 1973, Astrophysical Quantities, 3rd edition

Bertelli, G., Bressan, A., Chiosi, C., Fagotto, F., \& Nasi, E. 1994, A\&AS, 106, 275

Blaauw, A. \& Schmidt, M. (eds.), 1965 Galactic Structure, (Chicago: University of Chicago Press)

Boisse, P. 1990, A\&A, 228, 483

Bosma, A., Byun, Y. I., Freeman, K. C., \& Athanassoula, E. 1992, ApJ, 400, L21

Bruzual, A. G., Magris, C. G., \& Calvet, N. 1988, ApJ, 333, 673

Burstein, D., Haynes, M. P., \& Faber, S. M. 1991, Nature, 353, 515

Byun, Y. I. 1993, PASP, 105, 993

Byun, Y. I., Freeman, K. C., \& Kylafis, N. D. 1994, ApJ, 432, 114

Corradi, R. L. M., Beckman, J. E., \& Simonneau, E. 1996, MNRAS, 282, 1005

Davies, J. I. \& Burstein, D. (eds.) 1995, The Opacity of Spiral Disks, (Dordrecht: Kluwer Academic Publishers)

de Grijs, R. \& van den Kruit, P. C. 1996, A\&AS, 117, 19

de Jong, R. S. 1996, A\&A, 313, 377, astro-ph/9604010

Di Bartolomeo, A., Barbaro, R., \& Perinotto, M. 1995, MNRAS, 277, 1279

Disney, M. J., Davies, J. I., \& Phillips, S. 1989, MNRAS, 239, 939

Feast, M. W., Thackeray, A. D., \& Wesselink, A. J. 1960, MNRAS, 121, 337

Feast, M. \& Walker, A. R. 1987, ARA\&A, 25, 345

Fitzpatrick, E. L. 1985, ApJ, 299, 219

Gilmore, G., Wyse, R. F. G., \& Kuijken, K. 1989, ARA\&A, 27, 555

Giovanelli, R., Haynes, M. P., Salzer, J. J., Wegner, G., da Costa, L. N., \& Freudling, W. 1994, AJ, 107, 2036

Grieve, G. R. \& Madore, B. F. 1986, ApJS, 62, 427

Hill, R. J., Madore, B. F., \& Freedman, W. L. 1994, ApJ, 429, 192 
Hiltner, W. A. \& Johnson, H. L. 1956, ApJ, 124, 367

Hobson, M. P. \& Scheuer, P. A. G. 1993, MNRAS, 264, 145

Huizinga, J. E. \& van Albada, T. S. 1992, MNRAS, 254, 677

Isserstedt, J. 1975, A\&A, 41, 175

Johnson, H. L. \& Morgan, W. W. 1953, ApJ, 117, 313

Kuchinski, L. E. \& Terndrup, D. M. 1996, AJ, 111, 1073

Landolt, A. 1992, AJ, 104, 340

Massey, P., Lang, C. C., DeGioia-Eastwood, K., \& Garmany, C. D. 1995, ApJ, 438, 188

Oestreicher, M. O., Gochermann, J. \& Schmidt-Kaler, T. 1995, A\&AS, 112, 495

Oestreicher, M. O. \& Schmidt-Kaler, T. 1996, A\&AS, 117, 303

Peletier, R. F., Valentijn, E. A., Moorwood, A. F. M., Freudling, W., Knapen, J. H., \& Beckman, J. E. 1994, A\&AS, 108, 621

Prévot, L., Rousseau, J., \& Martin, N. 1989, A\&AS, 225, 303

Schmidt, M. 1959, ApJ, 129, 243

Straižys, V. 1992, Stellar Photometry (Tucson: Pachart)

Stetson, P. 1987, PASP, 99, 191

Tully, R. B. \& Fouqué, P. 1985, ApJS, 58, 67

Tully, R. B., Pierce, M. J., Huang, J. S., Saunders, W., Verheijen, M. A. W., \& Witchalls, P. L. 1997, in preparation

Valentijn, E. A. 1990, Nature, 346, 153

Westerlund, B. E. 1990, ARA\&A, 2, 29

Witt, A., Thronson Jr., H. A., \& Capuano Jr., J. M. 1992, ApJ, 393, 611

Zaritsky, D., Harris, J., \& Thompson, I., in press, astro-ph/9706206

Zaritsky, D., Schectman, S., \& Bredthauer, G. 1996, PASP, 108, 104 


\section{Figure Captions}

Fig. 1.- A $B-V, V$ Hess diagram from our LMC drift scan survey image. Over 1.1 million stars are represented. Each star is represented by a two-dimensional Gaussian with dimensions given by the observational uncertainties. The vertical sequence labeled $\mathbf{M S}$ is the main sequence. The area labelled $\mathbf{R C}$ is the red clump. The sequence labeled $\mathbf{R G}$ is the red giant branch. The vertical sequence labeled FG is a foreground population of Galactic stars (cf. 3 3.1). The box in the upper left represents the photometric limits used to isolate candidate OB stars (cf. \$3.1). The box in the lower right represents the photometric limits used to isolate red giant branch stars ( $c f .83 .3)$. The greyscale is logarithmic to emphasize low surface brightness features.

Fig. 2.- Q-color plots for candidate OB stars in our sample. The left panel plots the Johnson and Morgan $Q_{1}$ parameter, adapted to the extinction curve of the LMC. The right panel plots our $Q_{2}$ parameter. The lines are fits to the photometry of unreddened Galactic OB stars. The color excess, $E(B-V)$, of any star is the vertical distance between that star and the line.

Fig. 3.- The correlation of $E(B-V)$ as determined by the Johnson and Morgan $Q_{1}$ parameter with $E(B-V)$ as determined by our $Q_{2}$ parameter. The line represents $E(B-V)_{Q_{1}}=E(B-V)_{Q_{2}}$. The $r m s$ scatter about this line is 0.042 mag, while the mean propagated error on our reddening measurements is 0.039 mag.

Fig. 4.- Left: The uncorrected color-magnitude diagram for our candidate OB stars. Right: The same CMD, but corrected for interstellar reddening as outlined in the text. The open circles represent a population of stars that are apparently not $\mathrm{OB}$ main sequence stars. These stars are also visible in Figure 2 as those with $Q>0$.

Fig. 5.- Left: A histogram of $E(B-V)$ of OB stars as determined from the $Q$ parameters. The curve is a Gaussian representing a population of zero-reddening stars with the same propagated photometric errors as our data. The curve has been normalized to twice the number of observed OB stars with measured reddening values less than zero. Right: The histogram of $E(B-V)$, after correcting for foreground extinction as determined by Oestreicher et al. (1995). The curve is again the prediction of negative reddening values based on the propagated errors. There is now a slight excess of stars with negative LOSRV. 
This may be due to unresolved clumpiness in the foreground dust distribution.

Fig. 6. - The distribution of pseudo-LOSRV of red giant branch stars. The lack of highly-reddened stars is an indication that our photometric selection criterion for OB stars $(B-V<0.4 \mathrm{mag})$ is not biasing our results. These values of $E(B-V)$ are relative to the blue envelope of the red giant branch. The true zeropoint of this distribution is undetermined.

Fig. 7.- The positions on the sky of the 2069 OB stars in our sample. We attempt an interpolation of the reddening derived from these stars to all other stars in the frame.

Fig. 8.- (a) The interpolated reddening map of our LMC scan. We use this map to correct the photometry of non-OB stars in the image. (b) The map of the dispersion of assigned planar reddening values at each grid point. In this and the following figure, the greyscale is indicated by the vertical bar on the right.

Fig. 9.- (a) The interpolated reddening map, based on the foreground-corrected LOSRV. This map is a better indication of the internal structure of dust in the LMC. (b) The map of the dispersion of planar reddening values, for the foreground-corrected map.

Fig. 10.- Top Row: Four simulated dust distributions. The simulations show reddening variations with spatial wavelengths of, from left to right: 240 arcminutes, 60 arcminutes, 15 arcminutes, and 7.5 arcminutes. In each case, the amplitude of the reddening variations is 0.7 magnitudes. Center Row: The recovery of these simulations by our reddening interpolation algorithm. Comparison to Figure 7 illustrates that our resolution of small-scale structure is highly dependent on the surface density of OB stars. In the dense regions, we can resolve variations to better than 7.5 arcminutes. Bottom Row: The recovery by our interpolation routine of the 15 arcminute simulation, with amplitudes of, from left to right: $0.32,0.16,0.08$, and $0.04 \mathrm{mag}$ (the smallest amplitude corresponds to $1 \sigma)$. In each of these models, the pixel scale has been normalized to match the amplitude of the 0.32 mag model. Our amplitude limit $(\sim 0.08$ mag at these spatial wavelengths) is not highly dependent on the surface density of OB stars. In each of these simulations, the axes are the same as those in Figures 7, 8, and 9.

Fig. 11. - Comparison of the reddening of OB stars as determined by the $Q$ parameter analysis with the reddening of these stars as re-calculated by the 
interpolation routine. High LOSRV are not reproduced in the map because they are generally uncorrelated.

Fig. 12.- Line-of-sight geometry models. Top row: Plane-parallel models. Uniform foreground sheet (left), dust and stars uniformly mixed (center), and infinitesmally thin midplane dust sheet (right). Center row: Exponential disk models. $h_{s} / h_{d}=0.5,1.0,2.0$ (left to right). Bottom Row: Exponential disk, plus clumpy component. Exponential tail distribution (left) and secondary gaussian distribution (center). In the bottom right, we reproduce the foregroundcorrected LOSRV histogram, for reference.

Fig. 13. - LOSRV vs. Right Ascension for a slice of declination: $-67.5^{\circ}<$ $\delta<-67.2^{\circ}$. If the dust layer is smooth, then the reddening should indicate the relative depth of each star along the line-of-sight. The implied large extent of the far side of the LMC and the elongation of the OB associations (indicated with arrows) along the line-of-sight indicate that the dust layer must be clumpy.

Fig. 14. - The median difference between LOSRV pairs as a function of angular separation for: all OB stars (upper left), field OB stars (upper right), all low reddening $\mathrm{OB}$ stars (lower left), and low reddening field OB stars (lower right). In each case, the solid line is the correlation of our measured LOSRV, and the dashed line is the correlation of the population after randomly reshuffling the LOSRV. The differences are normalized such that the mean difference of the randomized population is unity. The plot indicates that there is coherence in the reddening values out to separations of 20 arcminutes. The anti-correlation at large separations may be due to a global gradient in the LOSRV across the field.

Fig. 15. - A high-resolution reddening map of the largest OB association in this section of the LMC. The higher resolution was achieved by using a higher density of map grid points, and by requiring six (instead of 20) LOSRV for each plane fit. These changes are justified by the high density of OB stars in this area. The cross marks the geometric centroid of the OB stars in this area $\left(5.21^{h}<\alpha<5.24^{h},-67.5^{\circ}<\delta<-67.3^{\circ}\right)$. Note that this does not correspond to the area of highest reddening, and that a high-reddening shell-like structure borders the area. 


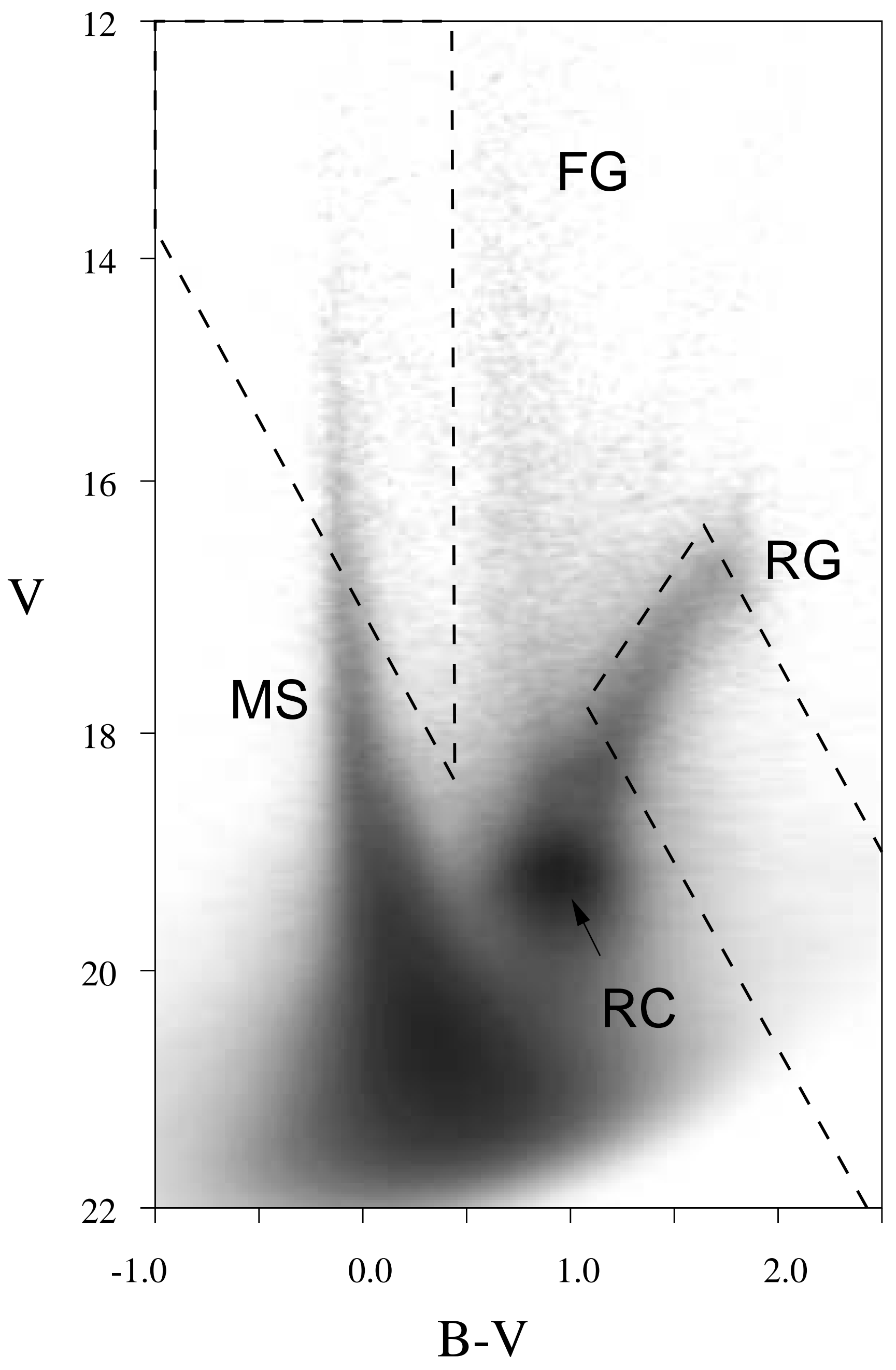




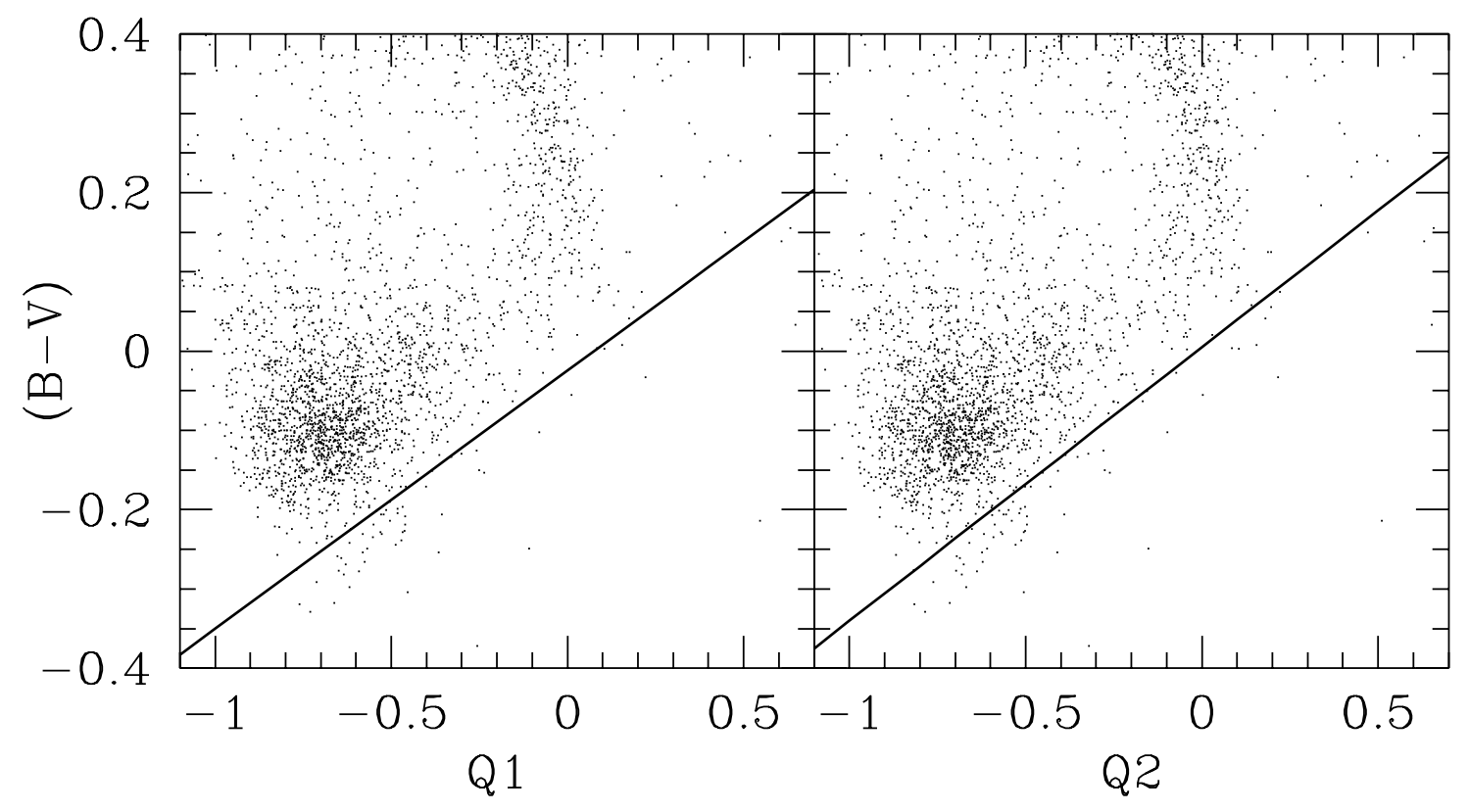




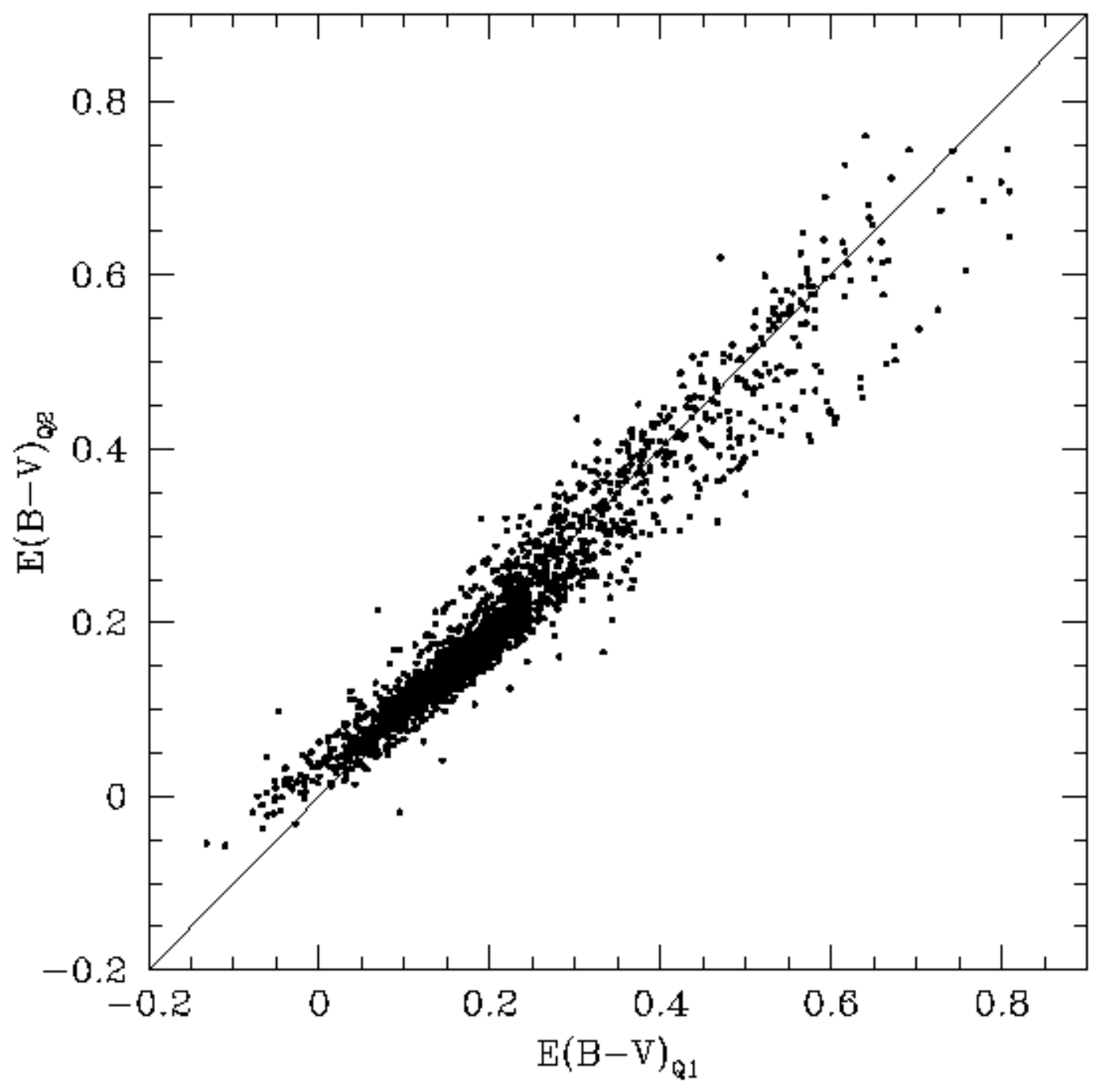




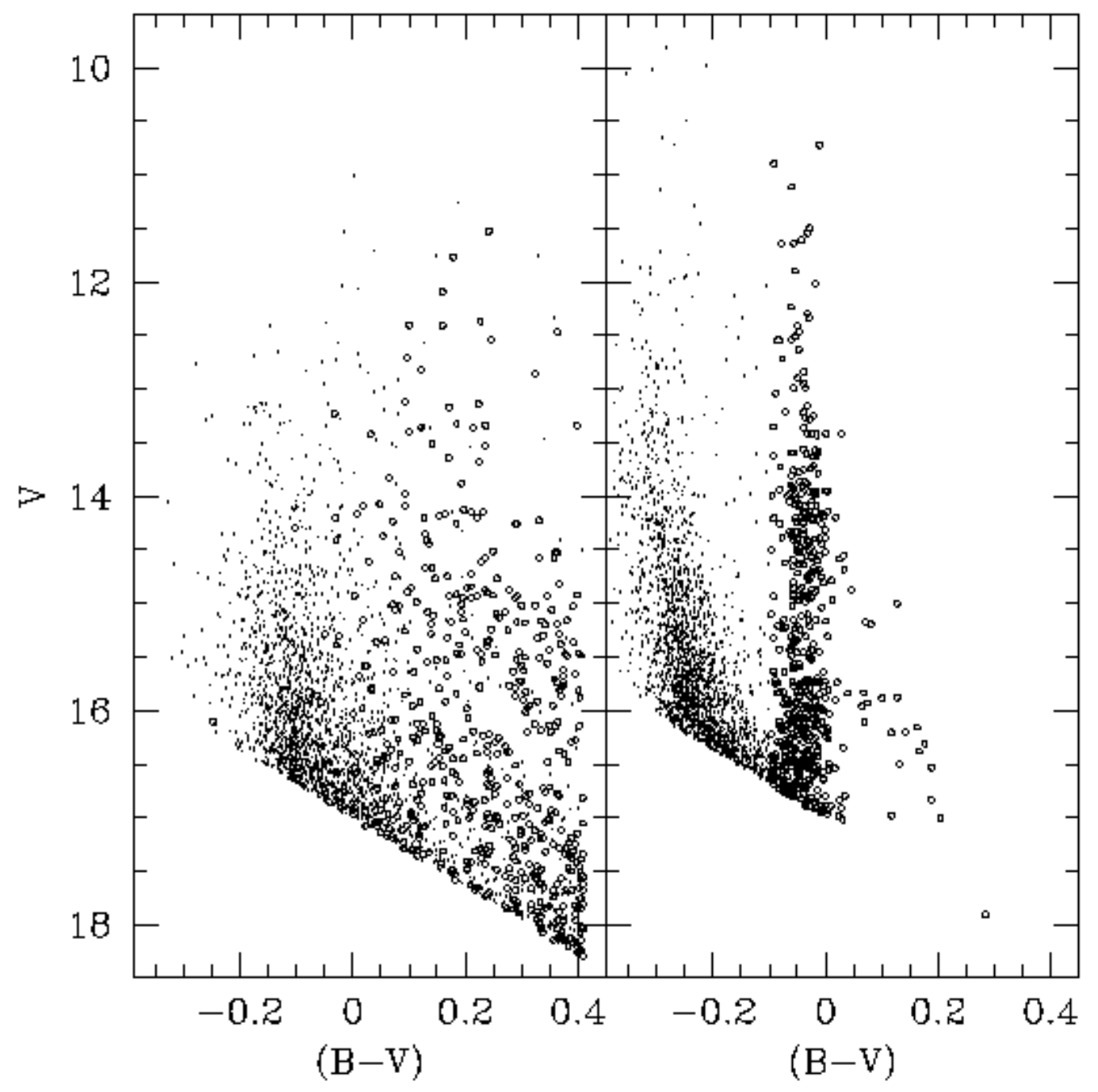




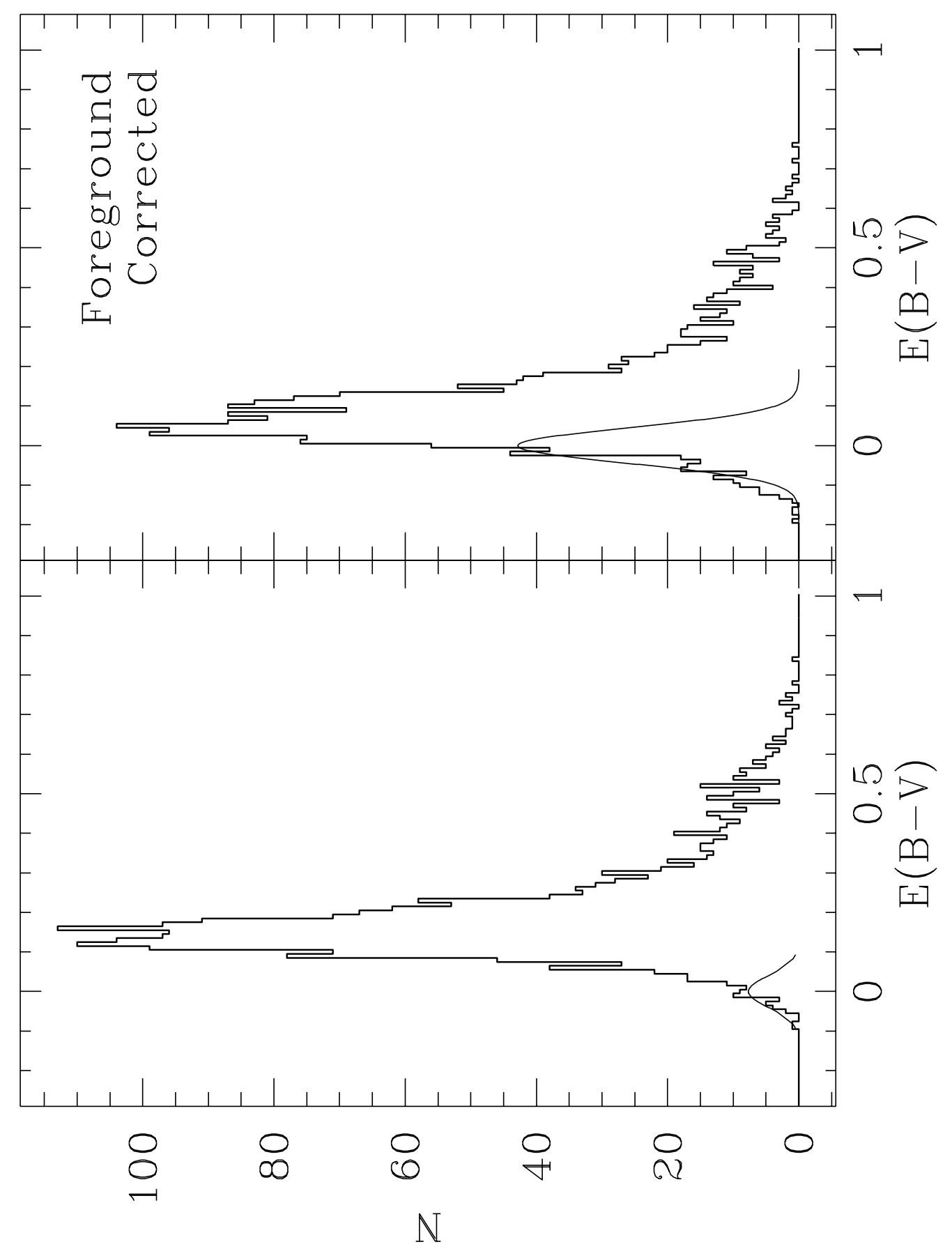




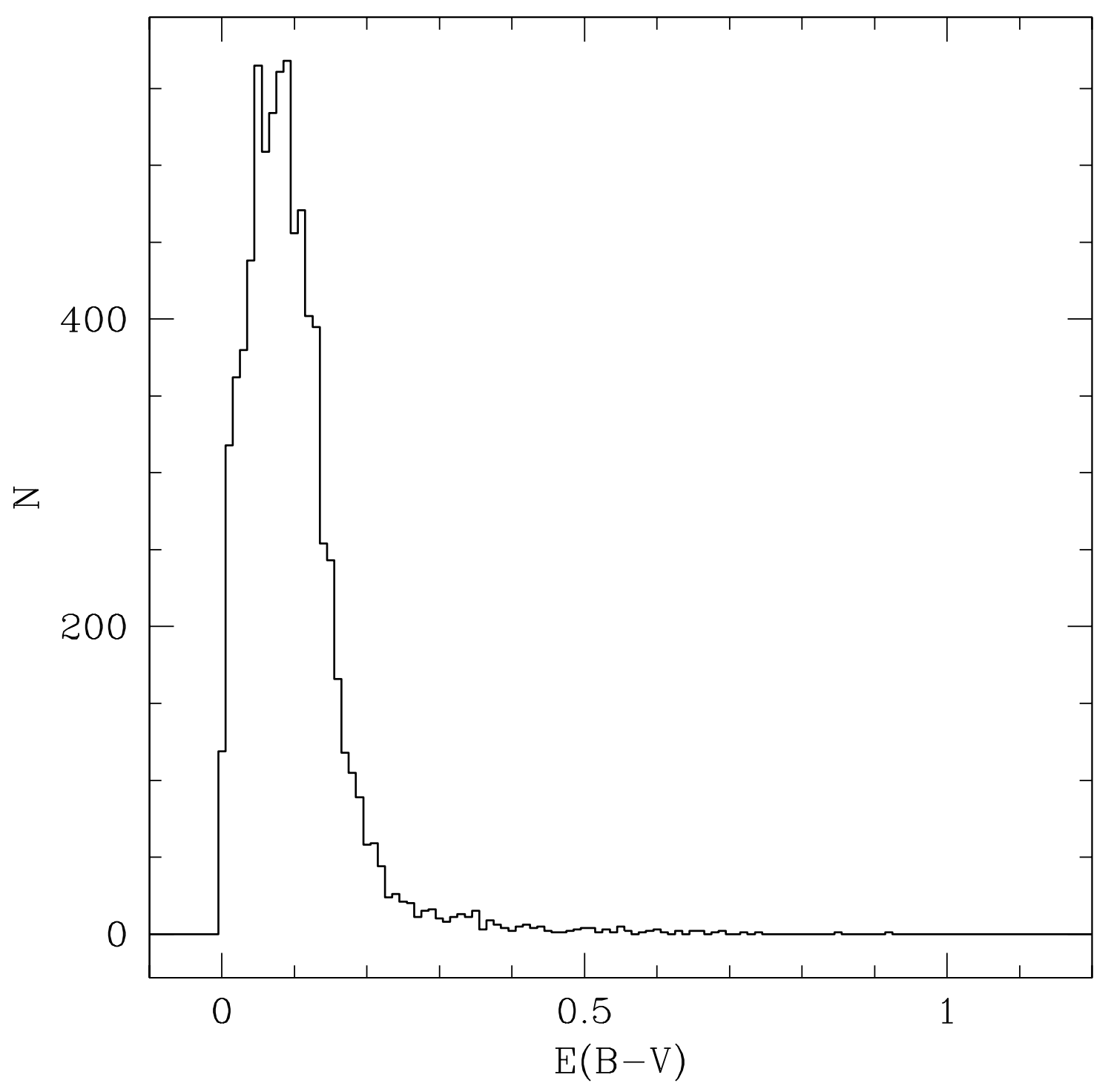




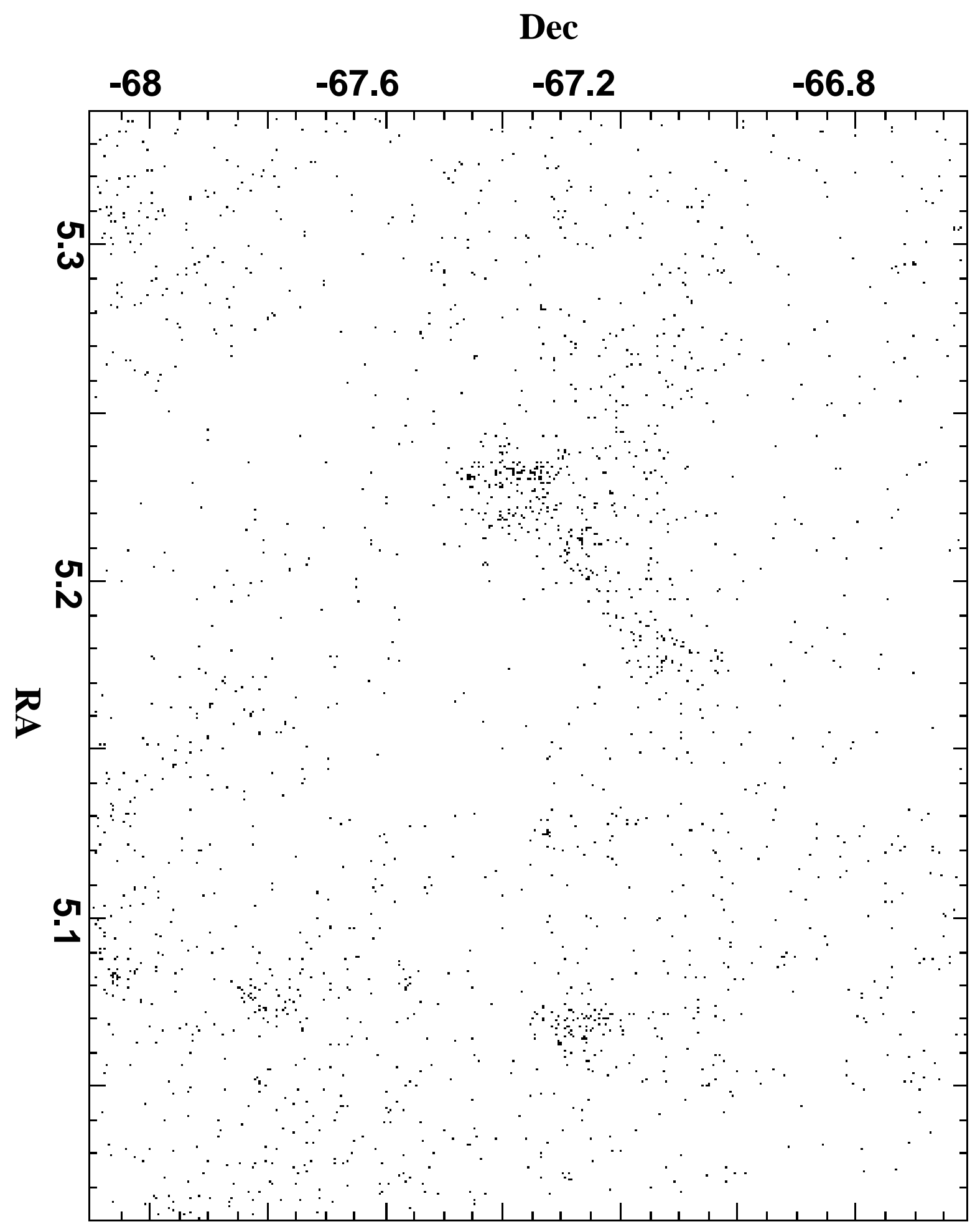




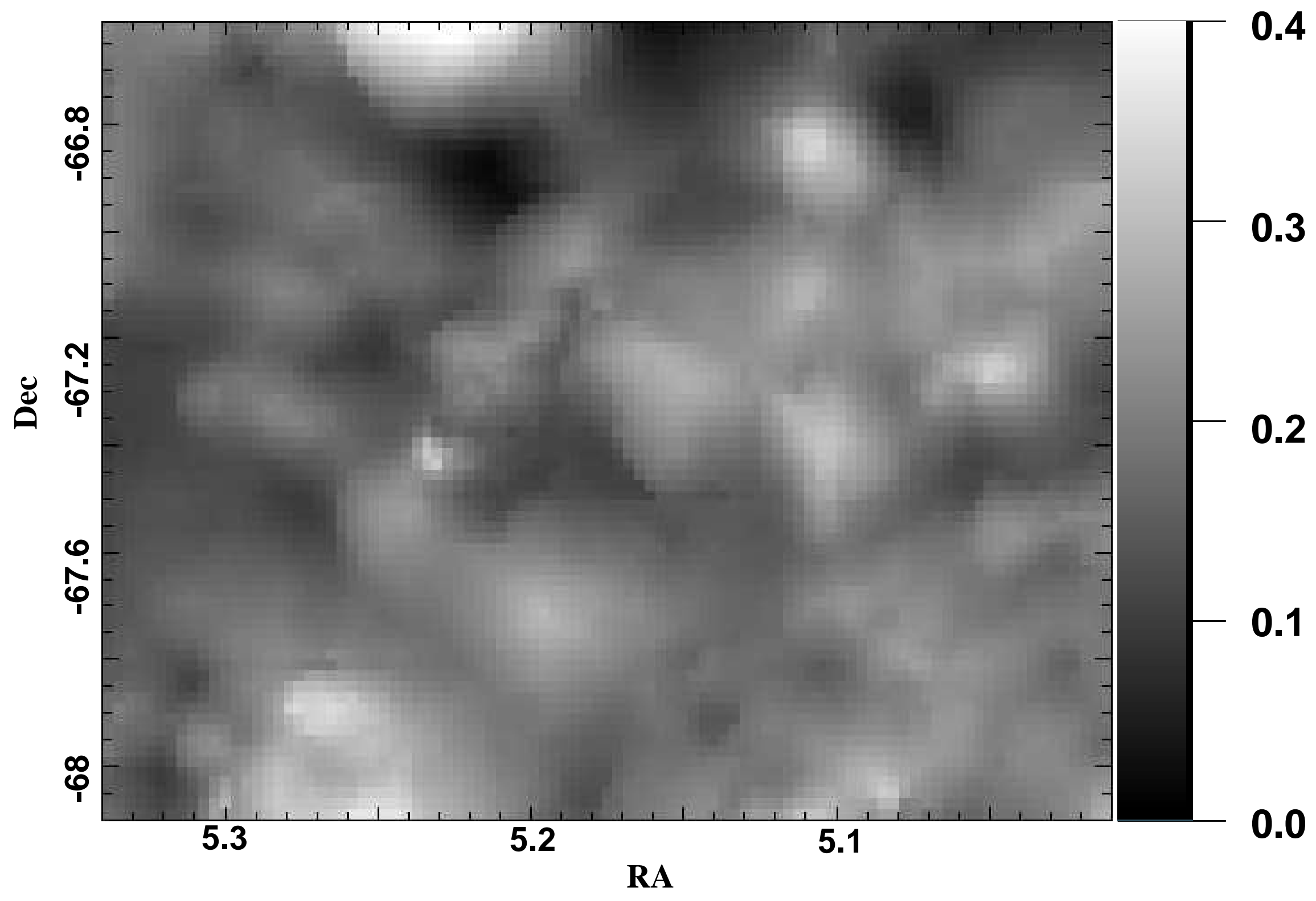




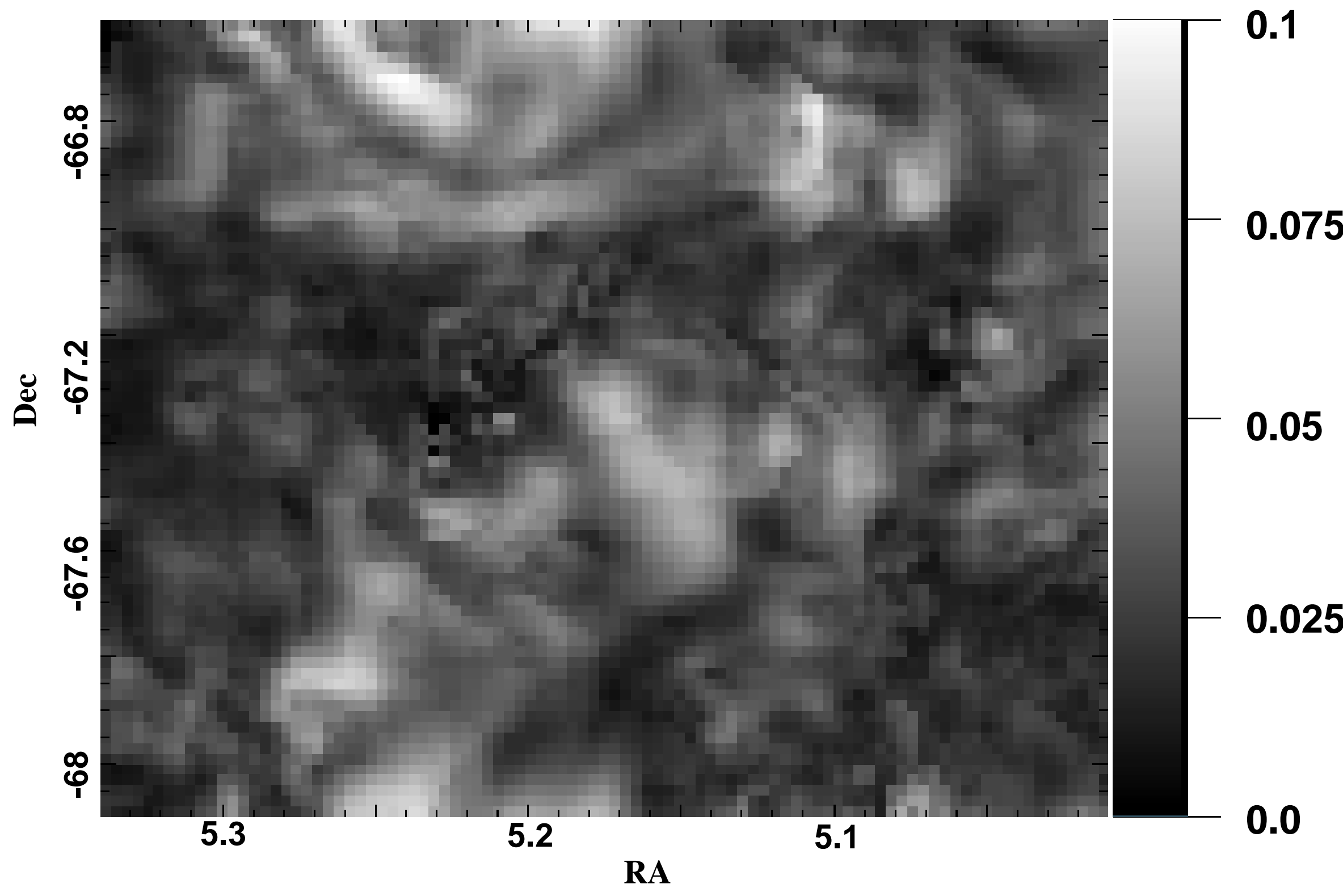




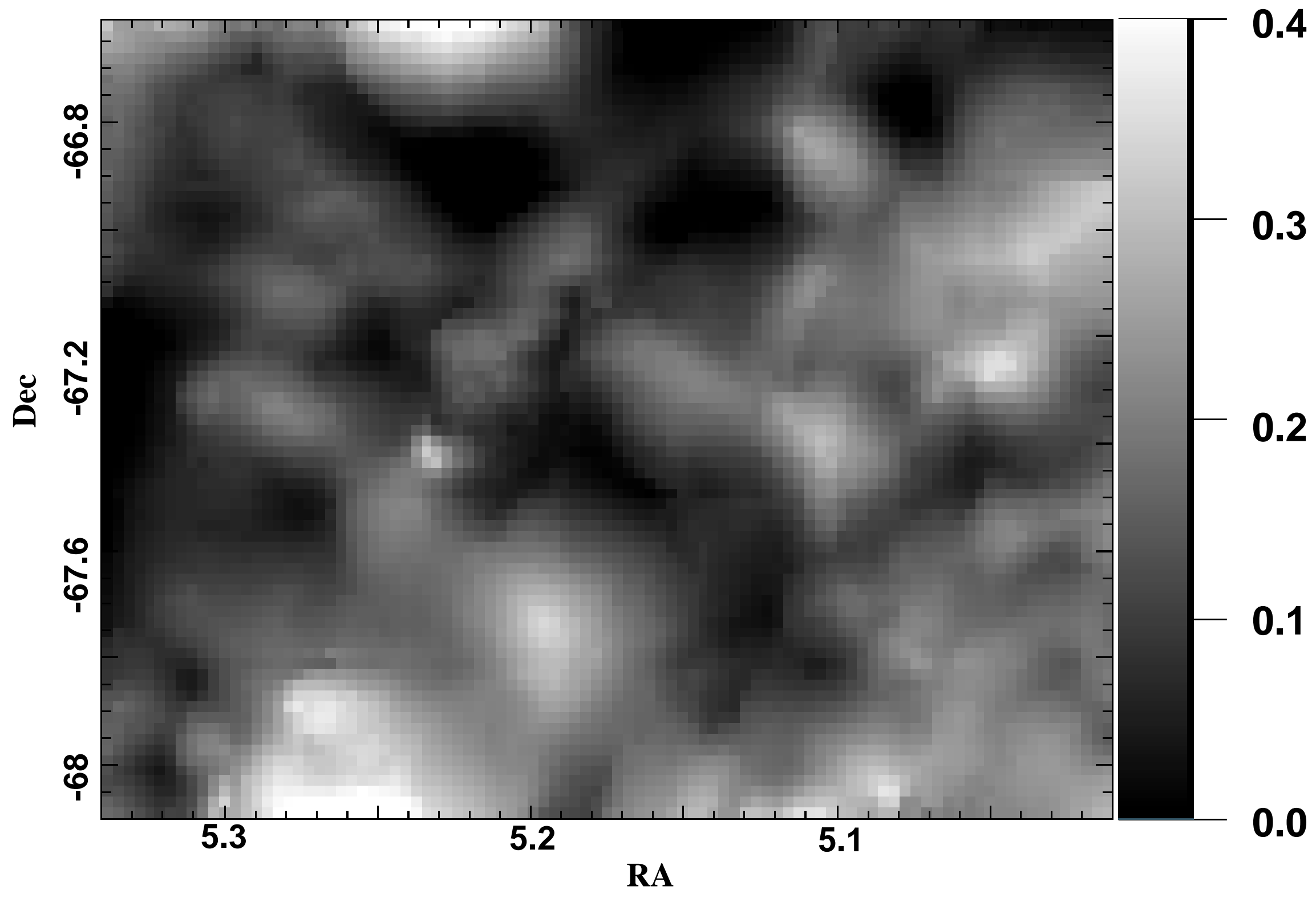




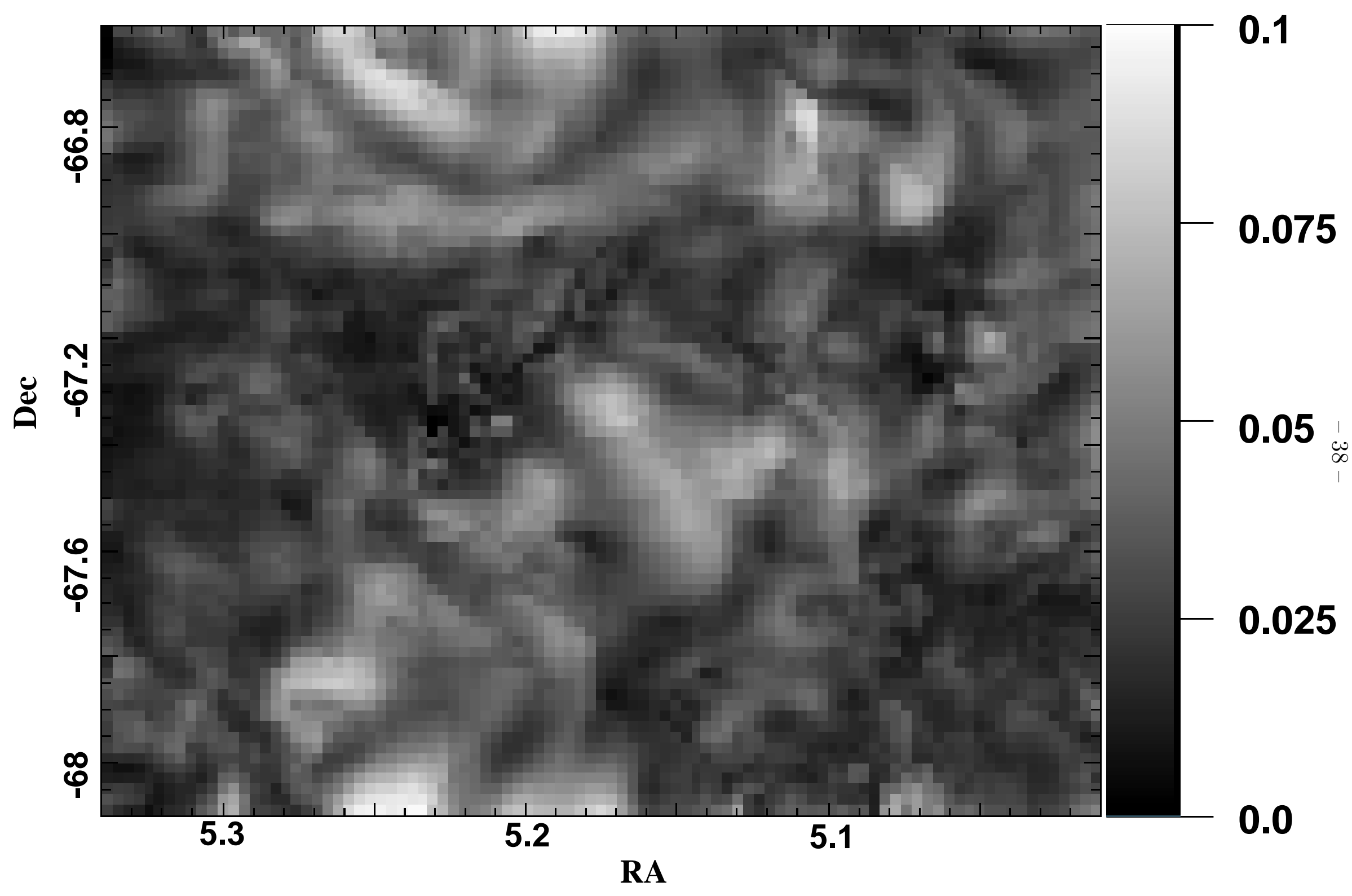



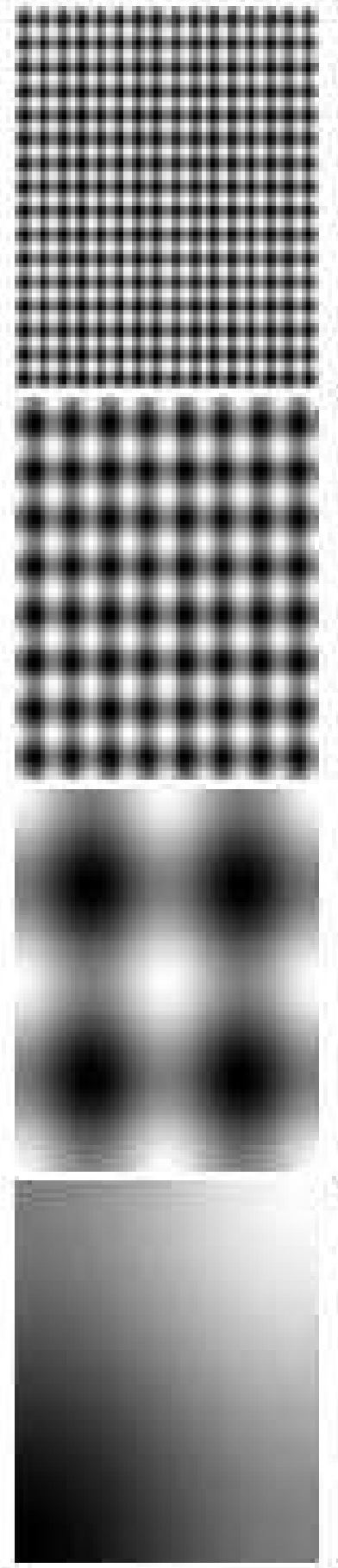
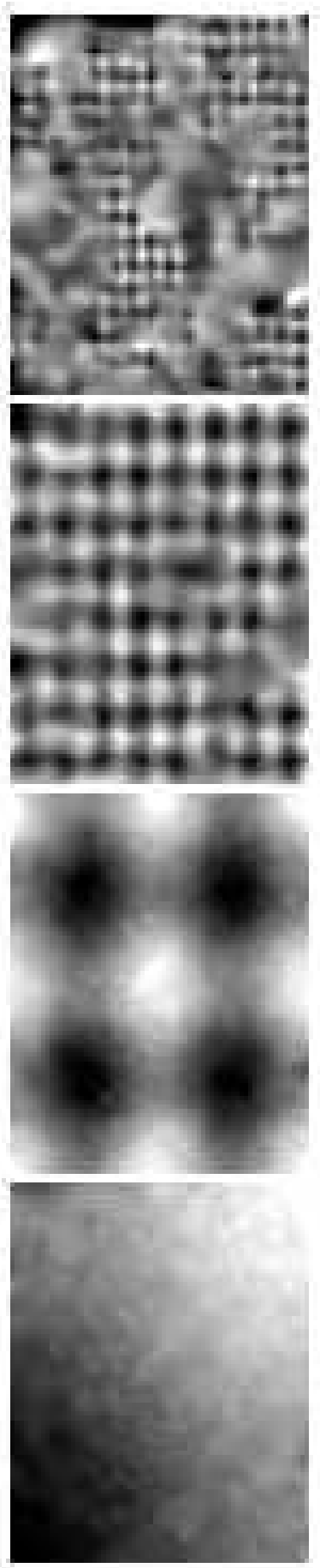
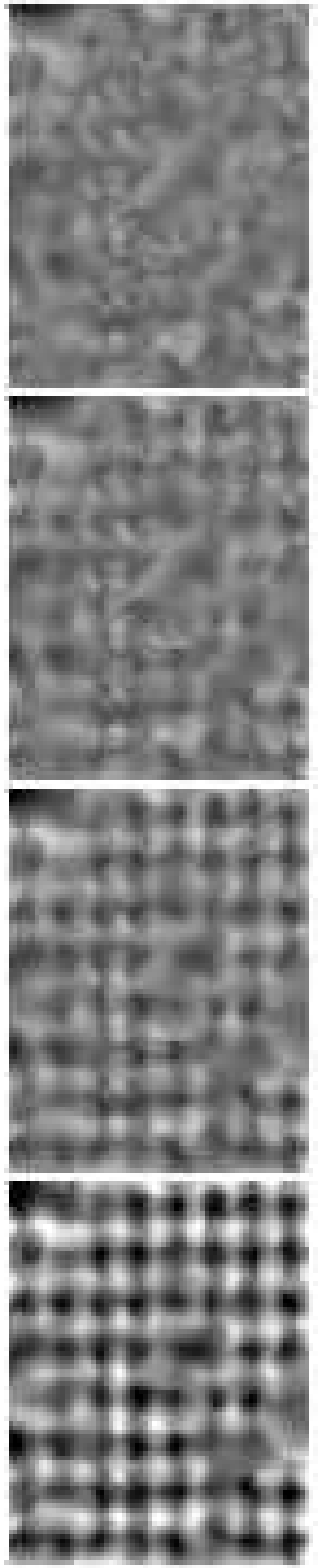


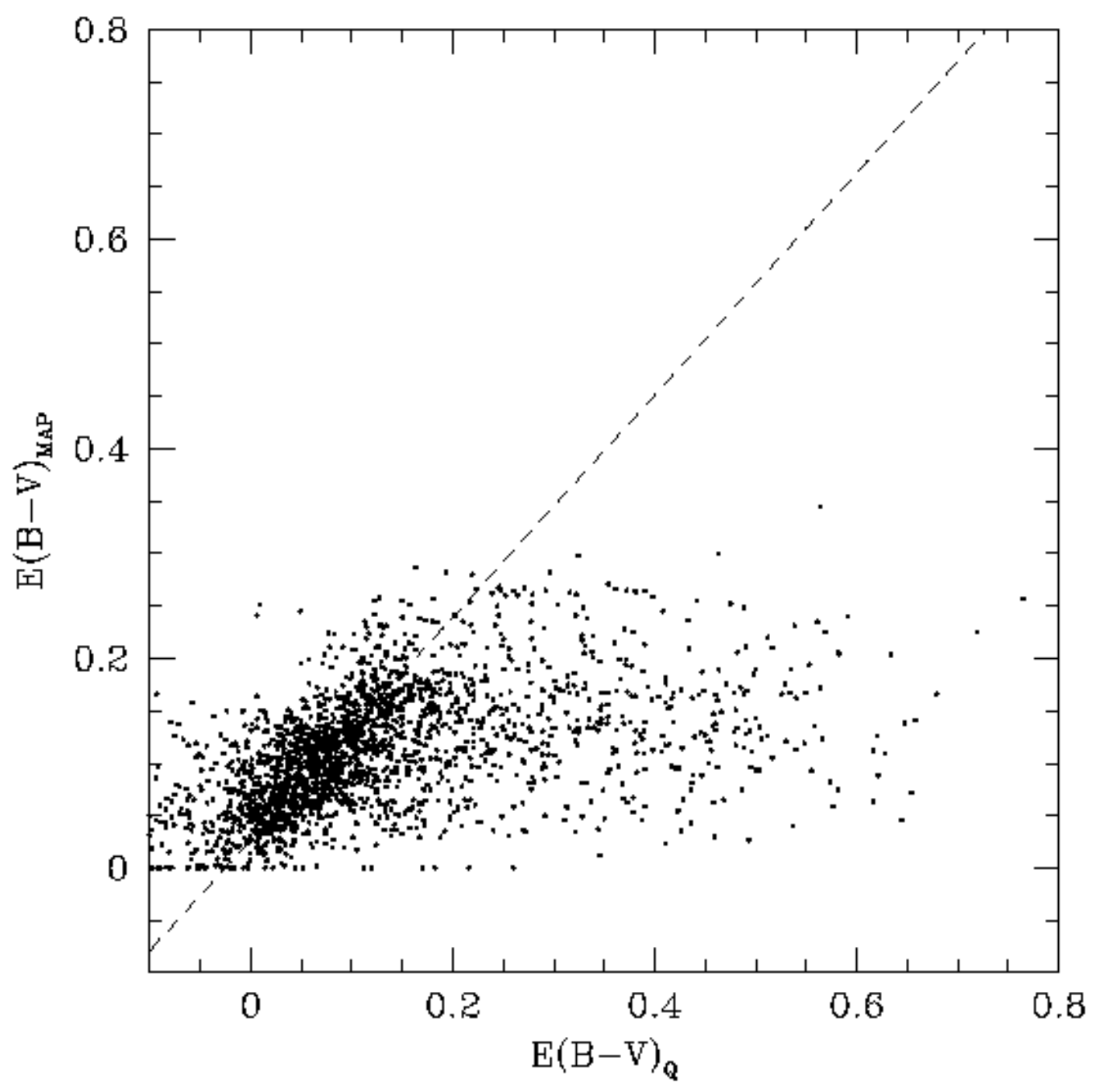




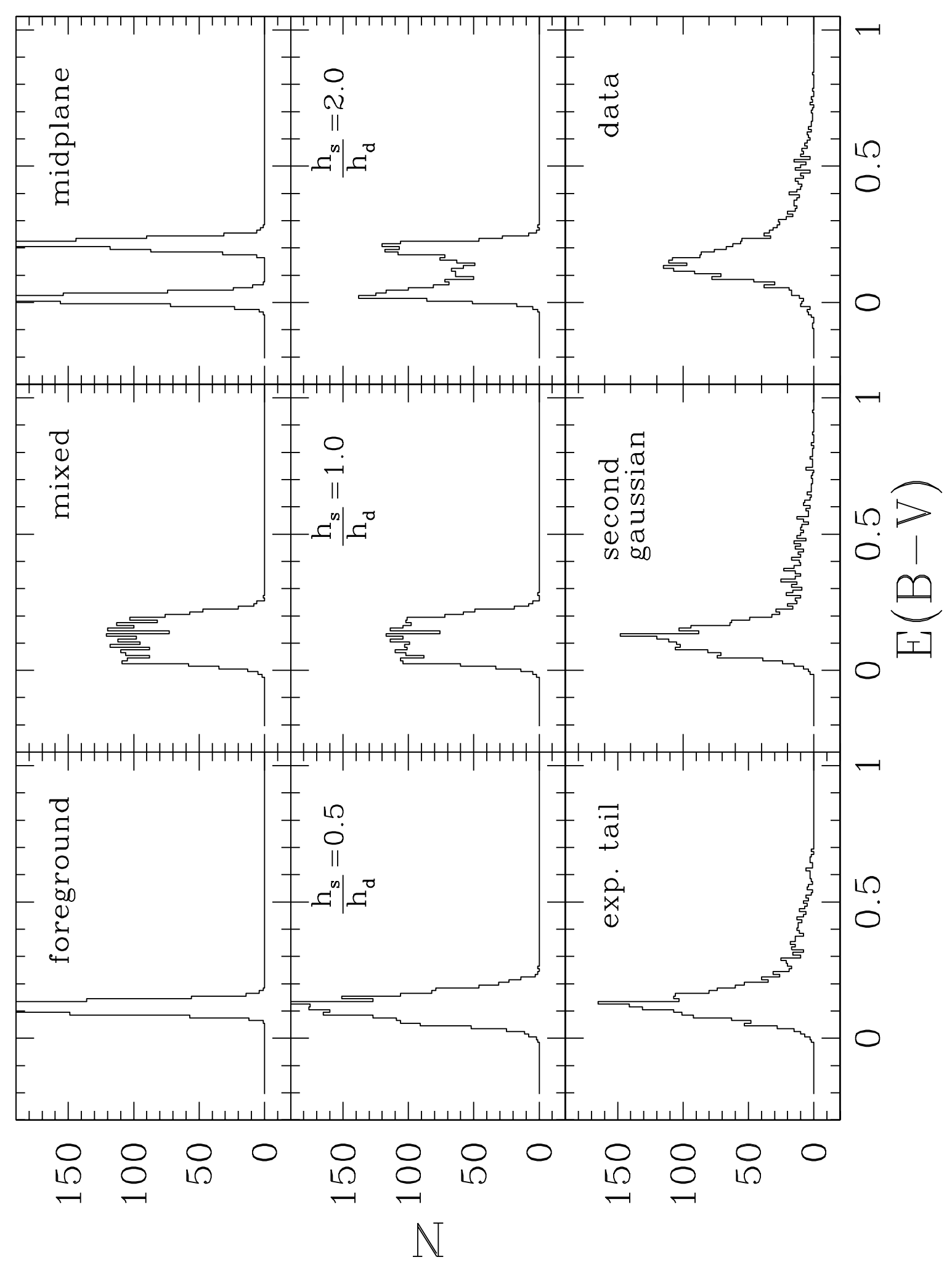




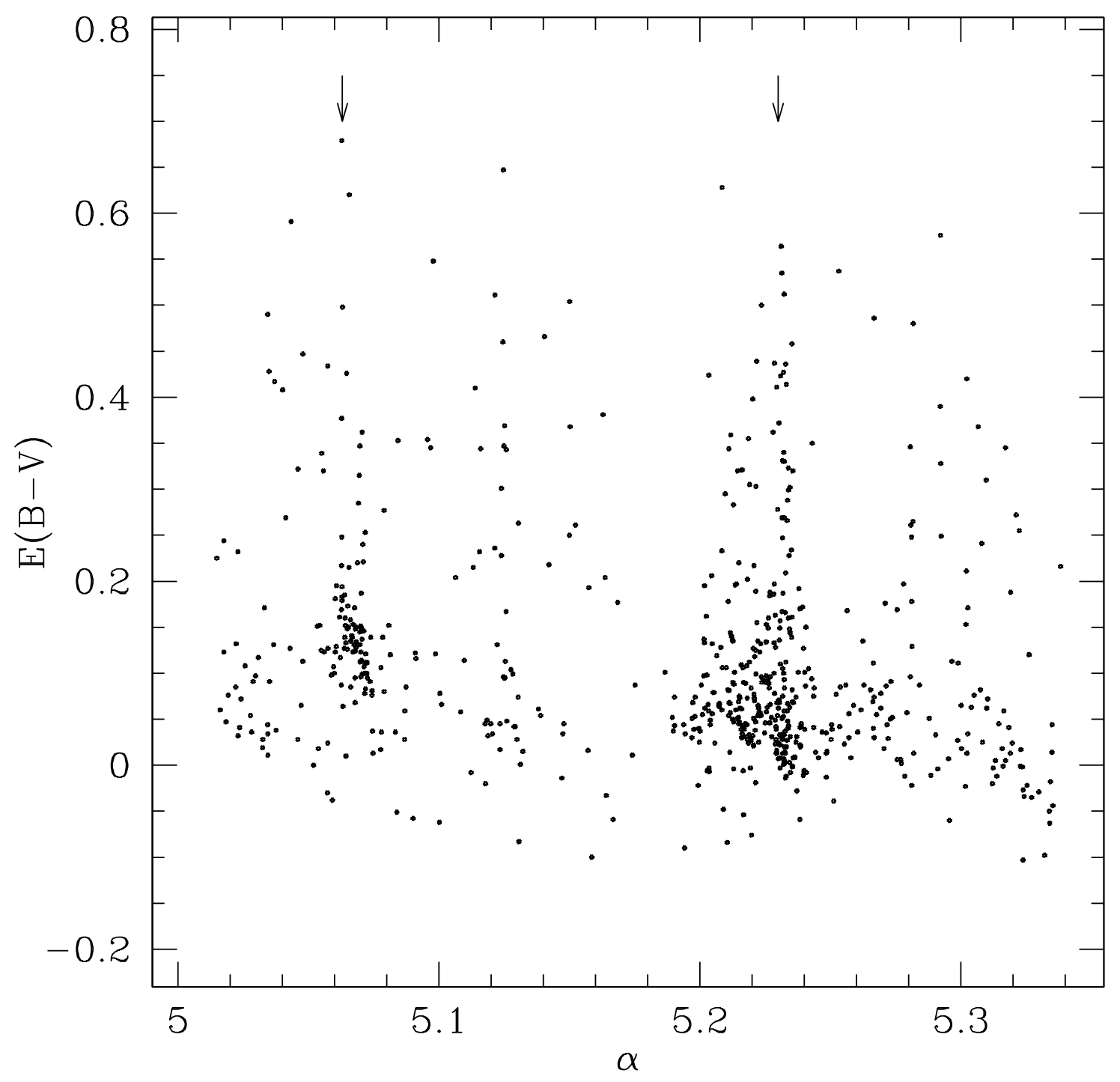




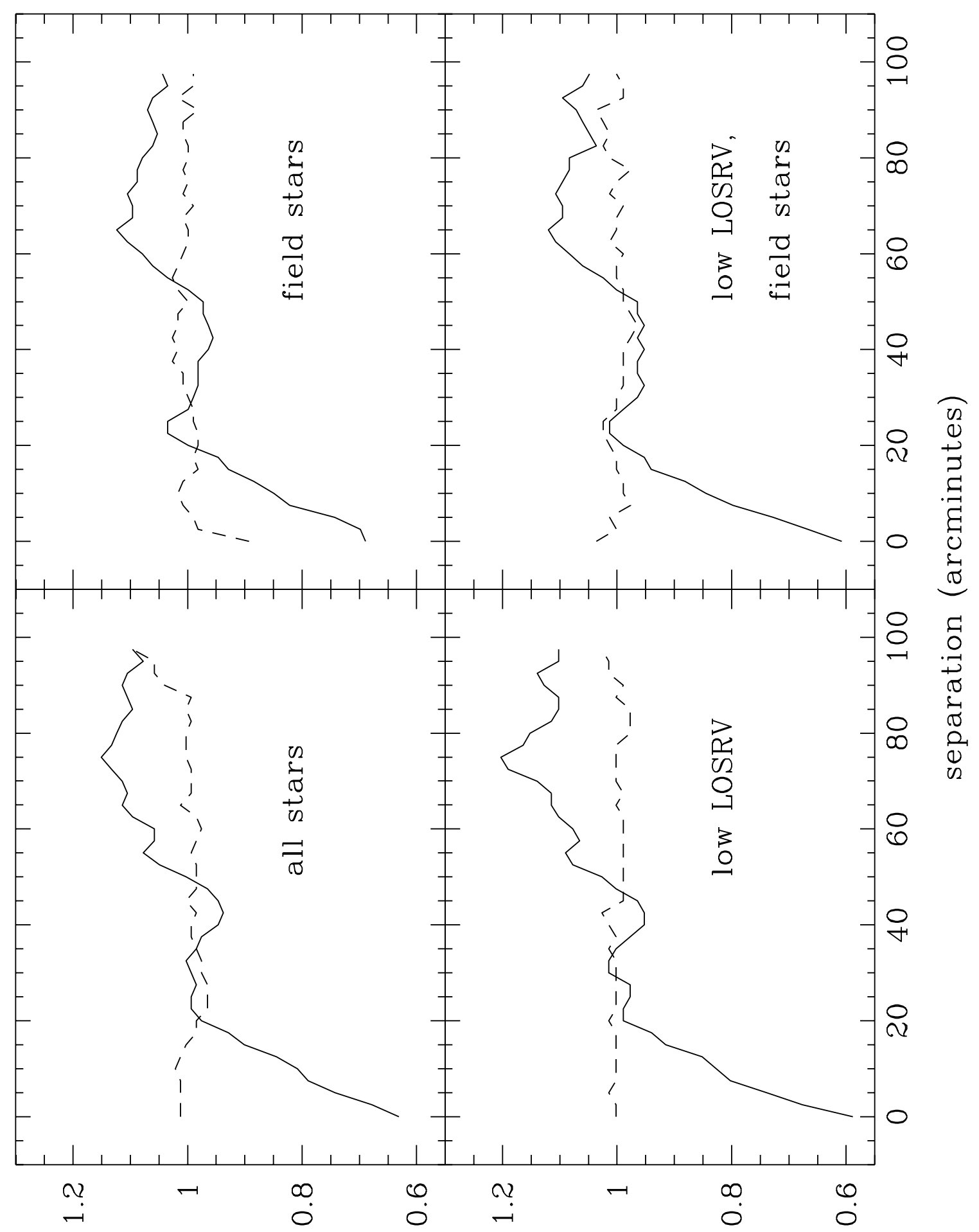




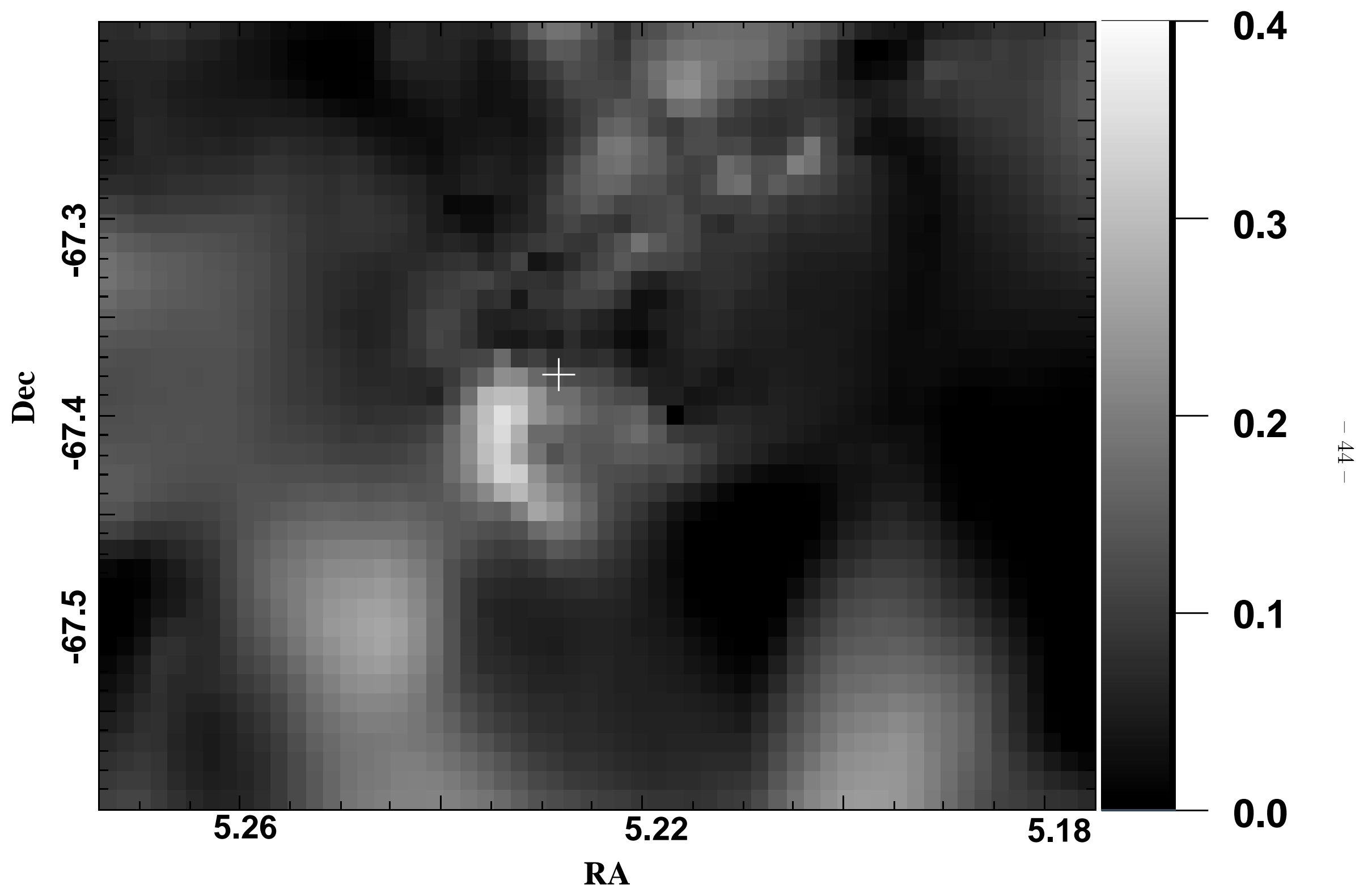

